\title{
KINEMATIC PROPERTIES AND STELLAR POPULATIONS OF FAINT EARLY-TYPE GALAXIES. II. LINE-STRENGTH MEASUREMENTS OF CENTRAL COMA GALAXIES
}

\author{
A. Matković ${ }^{1}$, R. Guzmán ${ }^{1}$, P. Sánchez-Blázquez ${ }^{2}$, J. Gorgas $^{3}$, N. Cardiel ${ }^{3}$, And N. Gruel ${ }^{1}$ \\ ${ }^{1}$ Astronomy Department, University of Florida, P.O. Box 112055, Gainesville, FL 32611, USA \\ ${ }^{2}$ Centre for Astrophysics, University of Central Lancashire, PR1 2HE, Preston, UK \\ ${ }^{3}$ Departamento de Astrofísica, Facultad de Físicas, Universidad Complutense de Madrid, Ciudad Universitaria, 28040 Madrid, Spain \\ Received 2008 February 11; accepted 2008 October 15; published 2009 February 12
}

\begin{abstract}
We present line-strength measurements for 74 early-type galaxies in the core of the Coma cluster reaching down to velocity dispersions, $\sigma$, of $30 \mathrm{~km} \mathrm{~s}^{-1}$. The index- $\sigma$ relations for our sample, including galaxies with $\sigma<100 \mathrm{~km}$ $\mathrm{s}^{-1}$ (low- $\sigma$ ), differ in shape depending on which index is used. We note two types of relations for the metallic indices: one showing a break in the slope around $\sim 100 \mathrm{~km} \mathrm{~s}^{-1}$ and another group with strong linear relations between an index and $\log \sigma$. We find no connection between the behavior of index $-\sigma$ relations with either $\alpha$ - or Fe-peak elements. However, we find indications that the relations are tighter for indices which do not depend on the microturbulent velocities of stellar atmospheres. We confirm previous results that low- $\sigma$ galaxies including $\mathrm{dE} / \mathrm{dS} 0 \mathrm{~s}$ are on average younger, less metal rich, and have lower $[\alpha / \mathrm{Fe}]$ in comparison with E/S0s. Our data show that these trends derived for high- $\sigma$ galaxies extend down to dE/dS0s. This is a factor of $\sim 2$ lower in $\sigma$ than previously published work. We confirm that the observed anticorrelation between age and metallicity for high- $\sigma$ galaxies is consistent with the effects of correlated errors. Low- $\sigma$ galaxies also show a similar relation between age and metallicity as a result of correlated errors. However, they are offset from this relationship so that, on average, they are less metal rich and younger than their high- $\sigma$ counterparts.
\end{abstract}

Key words: galaxies: abundances - galaxies: clusters: individual (Coma) - galaxies: dwarf - galaxies: elliptical and lenticular, $\mathrm{cD}$

Online-only material: color figures, machine-readable tables

\section{INTRODUCTION}

The "classical" view of the formation of massive early-type galaxies is that they formed relatively fast at early redshifts, and that they evolved passively. This is supported by tight scaling relations that these galaxies exhibit. However, the question of how their low-mass counterparts, dwarf early-type (dEs/dS0s) galaxies, formed remains unanswered.

One of the ways to investigate the star formation histories of early-type galaxies is through their line-strength indices which, when combined with stellar population models (SPMs), yield ages, and metallicities. However, the process of deriving star formation histories of galaxies by comparing line strengths with models is complicated by the degeneracy between age and metallicity (Worthey 1994), abundance ratio differences between the calibration stars used to calculate the models and the galaxy spectra, and strong Balmer lines which can be caused by either young stellar populations or an extended horizontal branch.

The most recent studies have incorporated iterative procedures and/or simultaneous fitting of as many indices as possible while deriving ages, metallicities, and relations between the line strengths and velocity dispersions $(\sigma)$ of early-type galaxies (Proctor et al. 2004; Thomas et al. 2005; Nelan et al. 2005; Denicoló et al. 2005; Sánchez-Blázquez et al. 2006a, 2006b). Despite these improved techniques and larger samples extending to lower luminosities, we still do not have a clear picture of the star formation histories of early-type galaxies, especially at the low-mass end.

It is well known that lower-luminosity early-type galaxies show a wider range in age than their more luminous counterparts (e.g., Caldwell 1983; Bender et al. 1993; Worthey \& Ottaviani 1997; Kuntschner \& Davies 1998; Poggianti et al. 2001a, 2001b; Caldwell et al. 2003). Some studies find that the lower-mass galaxies also display younger ages (Poggianti et al. 2001a, 2001b; Caldwell et al. 2003; Proctor et al. 2004; Thomas et al. 2005; Nelan et al. 2005), while others do not find this relation, or find that it depends on environment (Trager et al. 2000b; Kuntschner et al. 2001; Sánchez-Blázquez et al. 2006a). Similarly a number of studies show that lower-mass galaxies have lower metallicities (Brodie \& Huchra 1991; Poggianti et al. 2001a, 2001b; Kuntschner et al. 2001; Mehlert et al. 2003; Proctor et al. 2004; Nelan et al. 2005; Thomas et al. 2005; Sánchez-Blázquez et al. 2006b; Bernardi et al. 2006). Further, these galaxies display lower (closer to solar) abundance of $\alpha$ elements than the luminous elliptical galaxies (Es) which have an overabundance of $\alpha$-elements when compared to the values in the solar neighborhood (Gorgas et al. 1997; Jorgensen 1997; Trager et al. 2000b; Nelan et al. 2005; Thomas et al. 2005; Denicoló et al. 2005; Bernardi et al. 2006; Sánchez-Blázquez et al. 2006b). The lower values for the abundance ratio, $\alpha / \mathrm{Fe}$, for low-mass galaxies suggest that these galaxies have had more extended star formation histories than their massive counterparts.

The main goal of this project is to characterize internal kinematics and stellar populations of $\mathrm{dE} / \mathrm{dS} 0$ galaxies as a function of cluster environment. In Matković \& Guzmán (2005, hereafter Paper I) we described the internal kinematics of these galaxies in the dense central region of the Coma cluster. Here (Paper II) we investigate the properties of the underlying stellar populations of these galaxies. The following papers will examine the same properties of these galaxies in a region southwest of the cluster center, just outside the virial core, and will investigate how the location within the cluster affects these properties. Currently, there are only a few other studies which include $\mathrm{dE} / \mathrm{dS} 0$ galaxies in their samples. While these studies reach $\sigma \approx 50 \mathrm{~km} \mathrm{~s}^{-1}$ (Poggianti et al. 2001a; Caldwell et al. 2003; Nelan et al. 2005; 
Sánchez-Blázquez et al. 2006a), we present a statistically representative number of dE/dS0s in Coma reaching $\sigma=30 \mathrm{~km} \mathrm{~s}^{-1}$. In this paper, we investigate trends between the Lick/IDS line strengths and $\sigma$ and determine mean luminosity-weighted ages, metallicities, and $\alpha / \mathrm{Fe}$ ratios for a sample of 74 early-type galaxies in the central part of the Coma cluster. In Section 2, we describe our sample selection and our spectroscopic data; Section 3 describes the absorption line-strength measurements; in Section 4, we compare our measurements with other authors; in Section 5, we investigate the relations between different indices and $\sigma$; Section 6 describes the ages, metallicities, and $\alpha$-ratios.

\section{SAMPLE SELECTION AND SPECTROSCOPIC DATA}

We observed spectra of bright and faint early-type galaxies in two different environments within the Coma cluster, the central $20^{\prime} \times 20^{\prime}$ and a southwestern region, just outside the virial core $\left(\sim 1^{\circ}\right)$. In this paper, we discuss measurements of line-strength indices for 74 early-type galaxies (out of which 36 are $\mathrm{dE}$ / $\mathrm{dS} 0$ ) in the center of the Coma cluster, while the subsequent papers will also address a region outside the cluster virial core. We provided a full description of the sample selection and spectroscopic observations in Paper I. Here, we only present a summary of our observations.

For the $\mathrm{dE} / \mathrm{dS} 0$ sample selection we utilized photometry from WIYN's Mini-Mosaic imager and the Isaac Newton Telescope Wide Field Camera. We determined the following cutoffs from color-magnitude and color-color diagrams: $0.2<(U-B)<$ $0.6 \mathrm{mag}, 1.3<(B-R)<1.5 \mathrm{mag}$, and $M_{B} \geqslant-17.3 \mathrm{mag}$ (Ferguson \& Binggeli 1994) assuming a distance modulus for the Coma cluster of 35.078, $d=99 \mathrm{Mpc}$.

We obtained the follow-up spectroscopic data with the Hydra multifiber spectrograph on a WIYN $3.5 \mathrm{~m}$ telescope. The instrumental resolution was FWHM $=1.91 \AA$ which was sampled at $\sim 0.705 \AA \mathrm{px}^{-1}$. We used IRAF's “dohydra" package for data reduction. The diameter of the blue cable fiber was 3". 1 which corresponds to $\sim 1.45 \mathrm{kpc}$ at the distance of the Coma cluster $(d=99 \mathrm{Mpc})$.

In Paper I, we determined that $100 \%$ of the observed galaxies in our sample (down to $m_{B} \approx 19.2 \mathrm{mag}$ ) are consistent with being members of the Coma cluster as their recession velocities range between 4000 and 10,000 $\mathrm{km} \mathrm{s}^{-1}$ (Colless \& Dunn 1996). The luminosity of the sample spans $-20.3 \lesssim M_{B} \lesssim-15.8 \mathrm{mag}$ while the velocity dispersions are $30 \lesssim \sigma \lesssim 260 \mathrm{~km} \mathrm{~s}^{-1}$. All the spectra have a signal-to-noise ratio $(\mathrm{S} / \mathrm{N}) \geqslant 15$ per pixel (Paper I; also see Appendix B for $\mathrm{S} / \mathrm{N}$ determination).

Throughout this paper, we refer to galaxies fainter than $M_{B} \approx-18$ and with $\sigma<100 \mathrm{~km} \mathrm{~s}^{-1}$ as "faint," or low- $\sigma$ galaxies. This group includes the $36 \mathrm{dE} / \mathrm{dS} 0$ galaxies and six intermediate early-type galaxies. The remaining 32 objects with $\sigma \geqslant 100 \mathrm{~km} \mathrm{~s}^{-1}$ we refer to as bright or high- $\sigma$ galaxies.

In order to determine the luminosity-weighted ages and metallicities for the early-type galaxies in the Coma cluster we measure their spectral line-strength indices and compare them with SPMs by Thomas et al. (2003, hereafter TMB03).

\section{ABSORPTION-LINE-STRENGTH INDEX MEASUREMENTS}

We use the Lick/IDS system of indices (originally defined by Burstein et al. 1984; Gorgas et al. 1993; Worthey et al. 1994; Trager et al. 1998) as it covers a wide range in optical absorption lines among which are some of the most prominent features of early-type galaxies. Furthermore, the Lick/IDS system is also a basis for an extensive collection of stellar synthesis models, allowing one to derive ages and metallicities of galaxies.

We measured the line strengths for our 74 early-type galaxies with the software REDUCEME (Cardiel 1999), task INDEX. This software allows for careful determination of uncertainties in the index measurements as it uses Monte Carlo simulations (see Appendix A for more details). We were able to measure the following Lick/IDS indices: Ca4227, G4300, Fe4383, Ca4455, $\mathrm{Fe} 4531, \mathrm{C}_{2} 4668, \mathrm{H} \beta, \mathrm{Fe} 5015, \mathrm{Mg}_{1}, \mathrm{Mg}_{2}, \mathrm{Mg}_{b}, \mathrm{Fe} 5270$, and $\mathrm{Fe} 5335$ (Trager et al. 1998); and their extensions to higher order Balmer lines $\mathrm{H} \gamma_{A}$ and $\mathrm{H} \gamma_{F}$ (Worthey \& Ottaviani 1997). We also include the $[\mathrm{MgFe}]^{\prime}$ (as defined by TMB03) and $\langle\mathrm{Fe}\rangle$ (González 1993) indices since they closely measure metallicity and are common in the literature allowing for an easy comparison. We use these two indices with SPMs to predict luminosity-weighted ages, metallicities, and the $[\alpha / \mathrm{Fe}]-$ ratios.

The standard procedure for matching the Lick system of linestrength indices is to resample the data to the resolution of Lick/ IDS and to correct for the systematic differences between the indices of standard stars which were also observed by Lick. Additionally, one needs to correct the nebular emission and apply velocity dispersion and aperture corrections. We were not able to fully transform our data into the Lick/IDS system because we did not observe stars in common with the original stellar library (see below). Below, we describe the procedure that we followed.

1. Resampling to Lick/IDS resolution. We adjusted the spectral resolution of our data to match the resolution of the Lick/IDS 7-10 $\AA$ (FWHM) depending on the wavelength. This was done by broadening each galaxy spectrum by the quadratic difference between the Lick resolution and that of the observed galaxy:

$$
\sigma_{\text {broad }}=\sqrt{\sigma_{\text {Lick }}^{2}-\sigma_{\text {gal }}^{2}-\sigma_{\text {Instr }}^{2}},
$$

where $\sigma_{\text {broad }}$ is the amount to broaden the galaxy by (in $\left.\mathrm{km} \mathrm{s}^{-1}\right), \sigma_{\text {Lick }}$ is Lick/IDS resolution for a particular index (see Table 5 of Sánchez-Blázquez et al. 2006a), $\sigma_{\text {gal }}$ is the velocity dispersion of the galaxy, and $\sigma_{\text {Instr }}$ represents the instrumental resolution of our data. Note that $\sigma_{\mathrm{obs}}^{2}=\sigma_{\mathrm{gal}}^{2}+\sigma_{\text {Instr }}^{2}$.

2. Flux calibration. Since we lacked observations of flux calibration stars in our sample, we used the flux calibration of Sánchez-Blázquez et al. (2006a) who have eight galaxies in common with our sample. First, we obtained the response curves for the eight matching galaxies by dividing each galaxy spectrum by that of the flux-calibrated galaxy. Then, we fitted a polynomial function to each of the response curves and created a mean flux calibration curve. This curve is used to flux calibrate the line strengths, while the individual response curves are later used to calculate the flux-related uncertainties in the index measurements (see Appendix A).

3. Offsets to Lick/IDS standards. We did not observe stars in common with Lick/IDS. Furthermore, we only have four galaxies also observed by Lick/IDS (Trager et al. 1998) which is insufficient for determining any offsets between our data and the Lick/IDS system. We are, however, able to compare our data to other data sets in the literature (see Section 4).

4. Velocity dispersion corrections. The velocity dispersion measurements of our sample of Coma cluster galaxies are 
Table 1

Index Measurements

\begin{tabular}{|c|c|c|c|c|c|c|c|c|c|c|c|c|c|c|c|c|c|c|}
\hline GMP & $\mathrm{Ca} 4227$ & $\delta_{4227}$ & 4300 & 4300 & $\mathrm{H} \gamma_{A}$ & $\delta_{H \gamma A}$ & $\mathrm{H} \gamma_{F}$ & $\delta_{H \gamma F}$ & $\mathrm{Fe} 4383$ & $\delta_{4383}$ & $\mathrm{Ca} 4455$ & $\delta_{4455}$ & $\mathrm{Fe} 4531$ & $\delta_{4531}$ & $\mathrm{C}_{2} 4668$ & $\delta_{C_{2} 4668}$ & $\mathrm{H} \beta$ & $\delta_{H \beta}$ \\
\hline 478 & 0.972 & 124 & 4.413 & 214 & -3.346 & 246 & -0.298 & 147 & 817 & 31 & 3 & 2 & 2.473 & 234 & 2.134 & 0.450 & 2.359 & .143 \\
\hline 2489 & 1.211 & 077 & 460 & 131 & -5.220 & 74 & -1.052 & 98 & 814 & 90 & 1.189 & 97 & 3.271 & & 2 & 9 & .005 & 091 \\
\hline 2510 & 1.213 & 095 & 5.216 & 162 & -5.554 & 99 & -1.392 & 116 & 145 & $\delta$ & 1 & 7 & 1 & & & 6 & .927 & .105 \\
\hline 516 & 1.128 & 086 & 4.811 & 0.150 & -4.761 & 39 & -1.088 & 07 & 27 & 21 & 5 & 3 & 69 & 4 & 0 & & 1.570 & 120 \\
\hline 2529 & 1.406 & 0.079 & 4.618 & 0.141 & -3.547 & 0.195 & -0.421 & 14 & 3.915 & & 19 & 20 & 2.203 & 77 & & & 1.772 & .106 \\
\hline 2535 & 1.208 & 0.095 & & & & & & & & & & & & & & & 08 & .107 \\
\hline 2541 & 1.165 & 103 & 5.426 & & & & -1.660 & & 5.029 & & & & & & & & 1.495 & .114 \\
\hline 585 & 0.484 & 0.087 & 0 & 0.147 & -2.300 & & -0.056 & & 23 & & & & & & & & 2.642 & .105 \\
\hline 2603 & 0.665 & 0.076 & & 0.133 & & & & & 2.770 & & & & & & & & 2.740 & .114 \\
\hline 2654 & 1.147 & 0.095 & 5.689 & 0.160 & -5.897 & 0.195 & -1.637 & 0.112 & 5.038 & 0.216 & 1.217 & 0.111 & 3.146 & 0.161 & 6.261 & 0.413 & 1.682 & 0.109 \\
\hline
\end{tabular}

(This table is available in its entirety in a machine-readable form in the online journal. A portion is shown here for guidance regarding its form and content.)

described in Paper I. The velocity dispersion corrections are usually done by, first, broadening an individual galaxy spectrum to an effective resolution of $\sqrt{\left(\sigma_{\text {Lick }}^{2}+\sigma_{\text {gal }}^{2}\right)}$. Second, the index measurements are corrected for the extra broadening due to velocity dispersion of the galaxy $\left(\sigma_{\text {gal }}\right)$ via polynomial fitting. In our case, $\sigma_{\text {gal }}<\sigma_{\text {Lick }}$ for all but four galaxies in our sample. Therefore, we are able to apply the velocity dispersion corrections by broadening our spectra by $\sqrt{\left(\sigma_{\text {Lick }}^{2}-\sigma_{\text {gal }}^{2}\right)}$ and then measuring the indices. This approach also avoids introducing a source of uncertainty associated with velocity dispersion corrections which may affect the derived $I-\sigma$ relations (Kelson et al. 2006).

5. Aperture corrections. The galaxies in our sample are typically smaller than the $3^{\prime \prime}$ aperture of HYDRA fibers (Graham \& Guzmán 2003). Therefore, we found no need for aperture corrections. In fact, for most of our data, we are sampling the entire galaxy, rather than only its central parts. Since dE galaxies have flat velocity dispersion gradients (e.g., Pedraz et al. 1998), there should not be a significant change between the central and global measurements of these galaxies. Although we do not apply aperture corrections, we do recognize that a few massive galaxies in this sample are likely to be larger than the HYDRAspectrograph fibers in which case we are sampling their central regions only.

6. Emission corrections. It is well known that some earlytype galaxies have nebular emission which can contaminate measurements of certain line-strength indices. For example, nebular emission in the $\mathrm{H} \beta$ feature would cause the $\mathrm{H} \beta$ linestrength index to appear lesser in value which in turn would lead to the derivation of an older age for a given galaxy. We selected our sample via color-magnitude and color-color diagrams to minimize the presence of emission. We also checked the spectra for weak emission lines by coadding all the galaxies to enhance the $\mathrm{S} / \mathrm{N}$ of any weak emission, and dividing each galaxy by its template (Kuntschner et al. 2001). Finally, we used a method similar to that of Hammer et al. (2001) and Kuntschner et al. (2002) to determine whether any galaxy in our sample contained emission. From each galaxy spectrum, we subtracted its optimal template (a spectrum of a linear combination of template stars optimized to fit each galaxy spectrum, see Paper I for more details). This method revealed that two galaxies in our sample had $\mathrm{H} \beta$ and $\mathrm{O}$ III emission. We exclude these two galaxies, GMP 3733 and GMP 2516, from our analysis. We note that the low number of $\mathrm{dE} / \mathrm{dS} 0$ galaxies with emission is consistent with other studies in the Coma cluster (Sánchez-Blázquez et al. 2006a; Smith et al. 2008) and with dwarf galaxies having their gas removed when they enter the cluster environment.

A sample of our measurements is presented in Tables 1 and 2, while the full data set is in electronic format. These indices are at the Lick/IDS resolution, so they can be easily compared with other sources in the literature. The error in each index measurement includes uncertainties associated with the flux calibration and with the photon noise. For a more detailed description of the uncertainty measurements, see Appendix A.

\section{COMPARISON WITH THE LITERATURE}

Converting our data to the Lick/IDS system resolution allows a direct comparison to other data sets in the literature. We compare our line-strength measurements to that of Poggianti et al. (2001a, hereafter P01), Nelan et al. (2005, hereafter NFPS), and Smith et al. (2008, hereafter S08). Although these studies contain a significantly larger number of galaxies, our data are complementary to these samples as they include a larger number of low-mass galaxies $\left(\sigma \lesssim 100 \mathrm{~km} \mathrm{~s}^{-1}\right)$ with velocity dispersion measurements reaching as low as $\sigma \approx 30 \mathrm{~km} \mathrm{~s}^{-1}$ in Coma.

The comparison between the three literature samples and our data is shown in Figures 1-3. The offsets are presented in Table 3. The mean offsets are defined as a difference between the index measurement in this paper and the index measurement in P01, S08, or NFPS (N05) $\langle\Delta I\rangle=I_{\text {here }}-I_{\text {other }}$. We also calculated the error in the mean offset, standard deviation, and the standard deviation expected by the errors.

The offsets between our sample and S08 are small and not significant for the majority of the indices. The indices for which the offsets are significant are $\mathrm{Mg}_{1}, \mathrm{Mg}_{2}$, and Fe5270 with mean offsets of $-0.014 \pm 0.003,-0.018 \pm 0.004$, and $-0.334 \pm 0.127$, respectively. However, the scatter between the S08 sample and ours is large and cannot be explained by the errors. The indices Ca4227, G4300, $\mathrm{H} \gamma_{A}, \mathrm{H} \gamma_{F}$, and $\mathrm{Ca} 4455$ each have a scatter $\sim$ three times larger than the scatter expected by the errors, while the scatter is $\sim$ two times larger than the scatter due to the errors for the majority of the other indices. The large scatter in the comparison between the S08 index measurements and ours is dominated by a group of galaxies which are systematically offset in the $\mathrm{H} \beta$ plot (red open circles in Figure 1). Because our $\mathrm{H} \beta$ and $[\mathrm{MgFe}]^{\prime}$ indices for these galaxies cannot be reproduced by the SPMs, we exclude them from further analysis and revisit this issue in Section 6.1. However, once these galaxies are excluded from the analysis, 

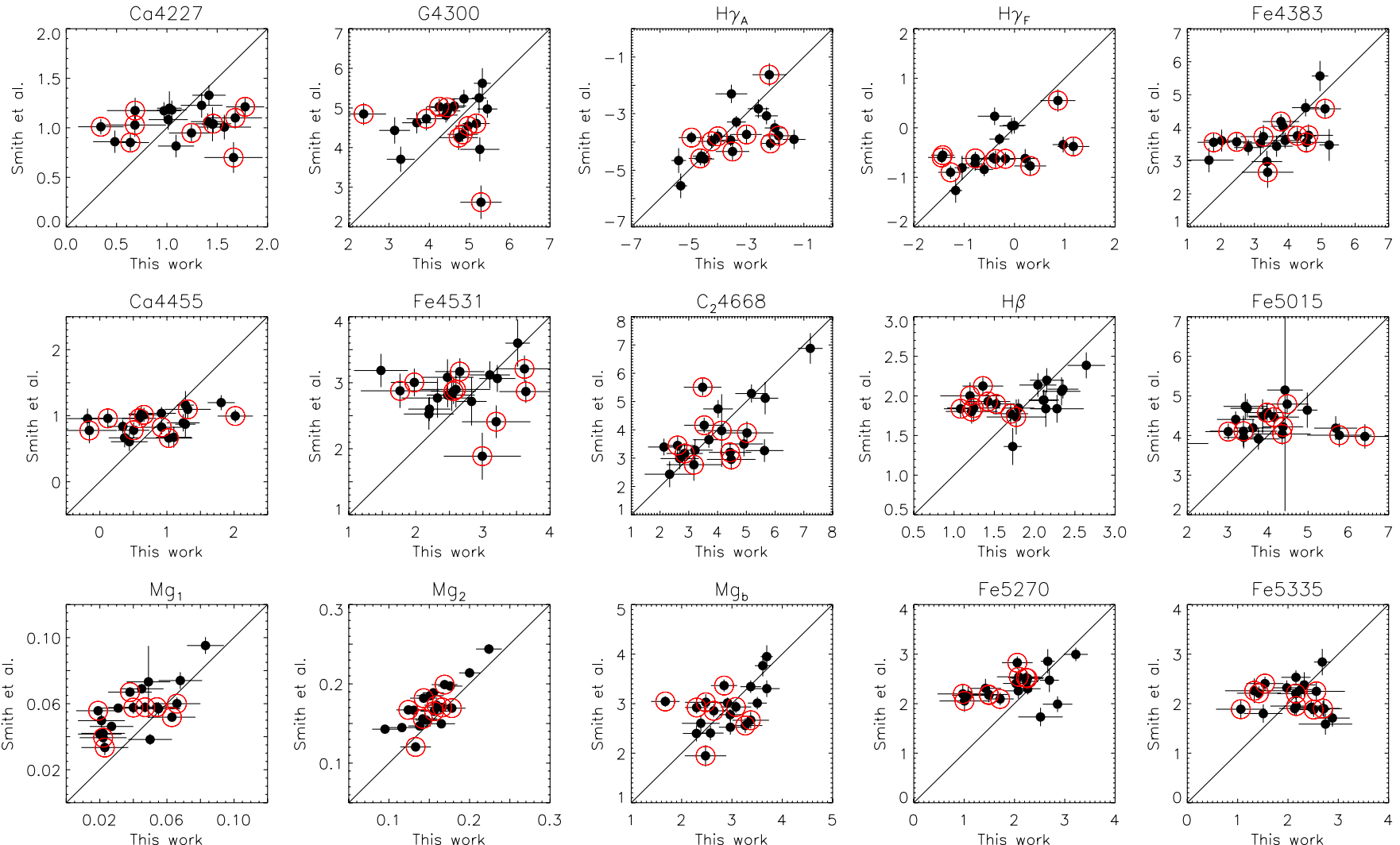

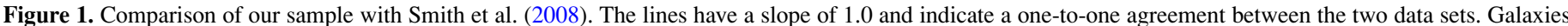
which do not fit on the model grids, the "off-grid" galaxies, are labeled as the red open circles and are discussed in Section 6.1.

(A color version of this figure is available in the online journal.)

Table 2

Index and Model Measurements

\begin{tabular}{cccccccccccccccccccc}
\hline \hline $\mathrm{GMP}$ & $\mathrm{Fe} 5015$ & $\delta_{5015}$ & $\mathrm{Mg}_{1}$ & $\delta_{\mathrm{Mg}_{1}}$ & $\mathrm{Mg}_{2}$ & $\delta_{\mathrm{Mg}_{2}}$ & $\mathrm{Mg}_{b}$ & $\delta_{\mathrm{Mg}_{b}}$ & $\mathrm{Fe} 5270$ & $\delta_{5270}$ & $\mathrm{Fe} 5335$ & $\delta_{5335}$ & $\mathrm{Age}$ & $\delta_{\mathrm{Age}}$ & {$[\mathrm{Z} / \mathrm{H}]$} & $\delta_{[\mathrm{Z} / \mathrm{H}]}$ & {$[\alpha / \mathrm{Fe}]$} & $\delta_{[\alpha / \mathrm{Fe}]}$ & $\mathrm{SNR}$ \\
\hline 2478 & 3.629 & 0.364 & 0.027 & 0.013 & 0.146 & 0.012 & 2.966 & 0.163 & 2.031 & 0.199 & 2.463 & 0.237 & 3.8 & 1.5 & -0.108 & 0.093 & 0.145 & 0.075 & 28 \\
2489 & 4.799 & 0.210 & 0.075 & 0.012 & 0.213 & 0.015 & 3.963 & 0.095 & 2.555 & 0.112 & 2.398 & 0.135 & 5.5 & 1.6 & 0.133 & 0.076 & 0.26 & 0.032 & 54 \\
2510 & 5.257 & 0.279 & 0.088 & 0.012 & 0.227 & 0.011 & 4.074 & 0.116 & 2.797 & 0.141 & 2.594 & 0.170 & 5.5 & 1.3 & 0.183 & 0.078 & 0.198 & 0.047 & 48 \\
2516 & 5.229 & 0.273 & 0.112 & 0.012 & 0.254 & 0.016 & 4.141 & 0.131 & 2.723 & 0.152 & 2.565 & 0.181 & $\ldots$ & $\ldots$ & $\ldots$ & $\ldots$ & $\ldots$ & $\ldots$ & 46 \\
2529 & 3.202 & 0.296 & 0.031 & 0.012 & 0.131 & 0.011 & 2.913 & 0.123 & 2.262 & 0.152 & 2.133 & 0.183 & 10.7 & 0.8 & -0.313 & 0.067 & 0.163 & 0.038 & 28 \\
2535 & 4.328 & 0.265 & 0.091 & 0.012 & 0.240 & 0.014 & 4.368 & 0.116 & 2.686 & 0.136 & 2.440 & 0.163 & 5.6 & 2.2 & 0.214 & 0.126 & 0.303 & 0.038 & 47 \\
2541 & 4.954 & 0.287 & 0.115 & 0.012 & 0.263 & 0.014 & 4.692 & 0.124 & 2.621 & 0.147 & 2.344 & 0.178 & 13.5 & 0.7 & 0.063 & 0.052 & 0.35 & 0.027 & 45 \\
2585 & 5.684 & 0.266 & 0.020 & 0.012 & 0.116 & 0.015 & 2.294 & 0.127 & 1.080 & 0.153 & 1.509 & 0.183 & 5.9 & 1 & -0.714 & 0.102 & 0.478 & 0.079 & 23 \\
2603 & 4.875 & 0.299 & 0.020 & 0.012 & 0.115 & 0.012 & 2.355 & 0.133 & 2.248 & 0.159 & 2.353 & 0.192 & 2 & 0.7 & $\ldots$ & $\ldots$ & $\ldots$ & $\ldots$ & 39 \\
2654 & 4.671 & 0.255 & 0.101 & 0.012 & 0.236 & 0.015 & 4.042 & 0.113 & 2.710 & 0.131 & 2.437 & 0.158 & 10.1 & 2.5 & 0.037 & 0.077 & 0.2 & 0.042 & 51 \\
\hline
\end{tabular}

(This table is available in its entirety in a machine-readable form in the online journal. A portion is shown here for guidance regarding its form and content.)

we no longer have a statistically significant number of galaxies in common with the S08 sample for comparison.

We find that the mean offsets between our index measurements and those of Poggianti et al. (2001a) are small and in most cases insignificant. However, $\mathrm{Ca} 4455, \mathrm{Fe} 5015$, and $\mathrm{Mg}_{1}$ have non-negligible offsets, $-0.259 \pm 0.116,-0.652 \pm 0.208$, and $-0.012 \pm 0.006$, respectively. We note that some indices may be offset because our indices are not fully transformed to the Lick/IDS system such as the P01 sample. Similarly to the S08 data, the scatter in the indices when comparing our data with P01 is large for most indices, except for $\mathrm{Mg}_{1}$ and $\mathrm{Mg}_{2}$. Furthermore, the large scatter between these two data sets cannot be explained by the errors. The discrepancy between the measured scatter and the scatter due to the errors is the largest for G4300, $\mathrm{H} \gamma_{A}, \mathrm{H} \gamma_{F}$, and $\mathrm{Mg}_{1}$.
On the other hand, the NFPS index measurements are in good agreement with ours. Among the atomic indices only Ca4227, Fe4531, and Fe5015 have significant offsets: $-0.207 \pm 0.047$, $-0.152 \pm 0.066$, and $-0.313 \pm 0.102$, respectively, while the rest of the indices are consistent between the two studies with a small scatter. Most noticeably, ours and the NFPS indices are in very good agreement in the $\mathrm{Mg}$ indices after a systematic offset in $\mathrm{Mg}_{1}$ of $-0.023 \pm 0.002$ and in $\mathrm{Mg}_{2}$ of $-0.017 \pm 0.002$ is applied. The NFPS data are not flux calibrated which would mostly affect the molecular indices such as the $\mathrm{Mg}_{1}$ and $\mathrm{Mg}_{2}$ and may explain why there is an offset between the data sets for these two indices. However, the scatter in these two molecular indices is remarkably small ( $0.01 \mathrm{mag}$ for both). The scatter in the remaining indices is also consistent within the errors in both studies. 

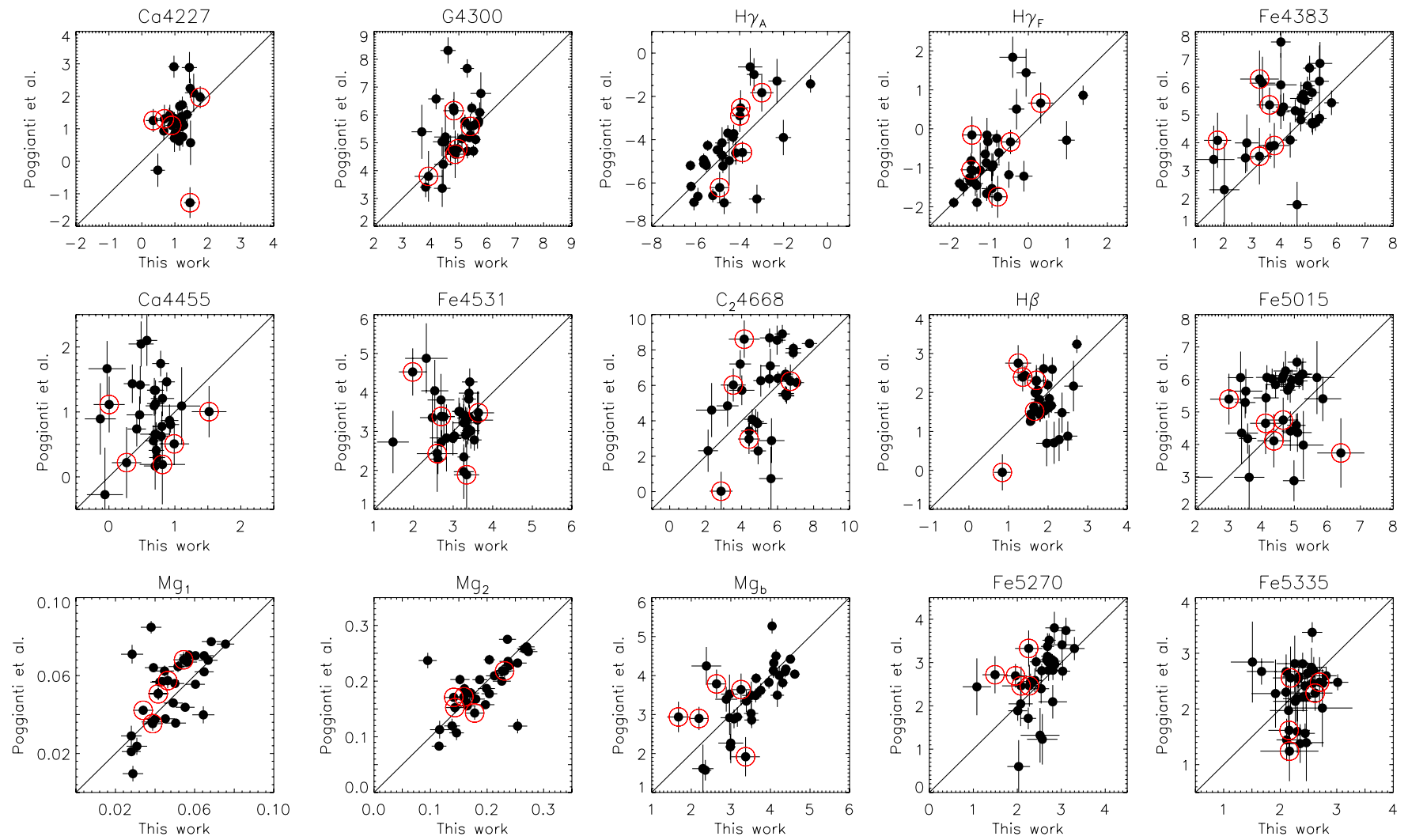

Figure 2. Comparison of our sample with Poggianti et al. (2001a) represented as the solid circles. The symbols and lines are the same as in Figure 1. (A color version of this figure is available in the online journal.)
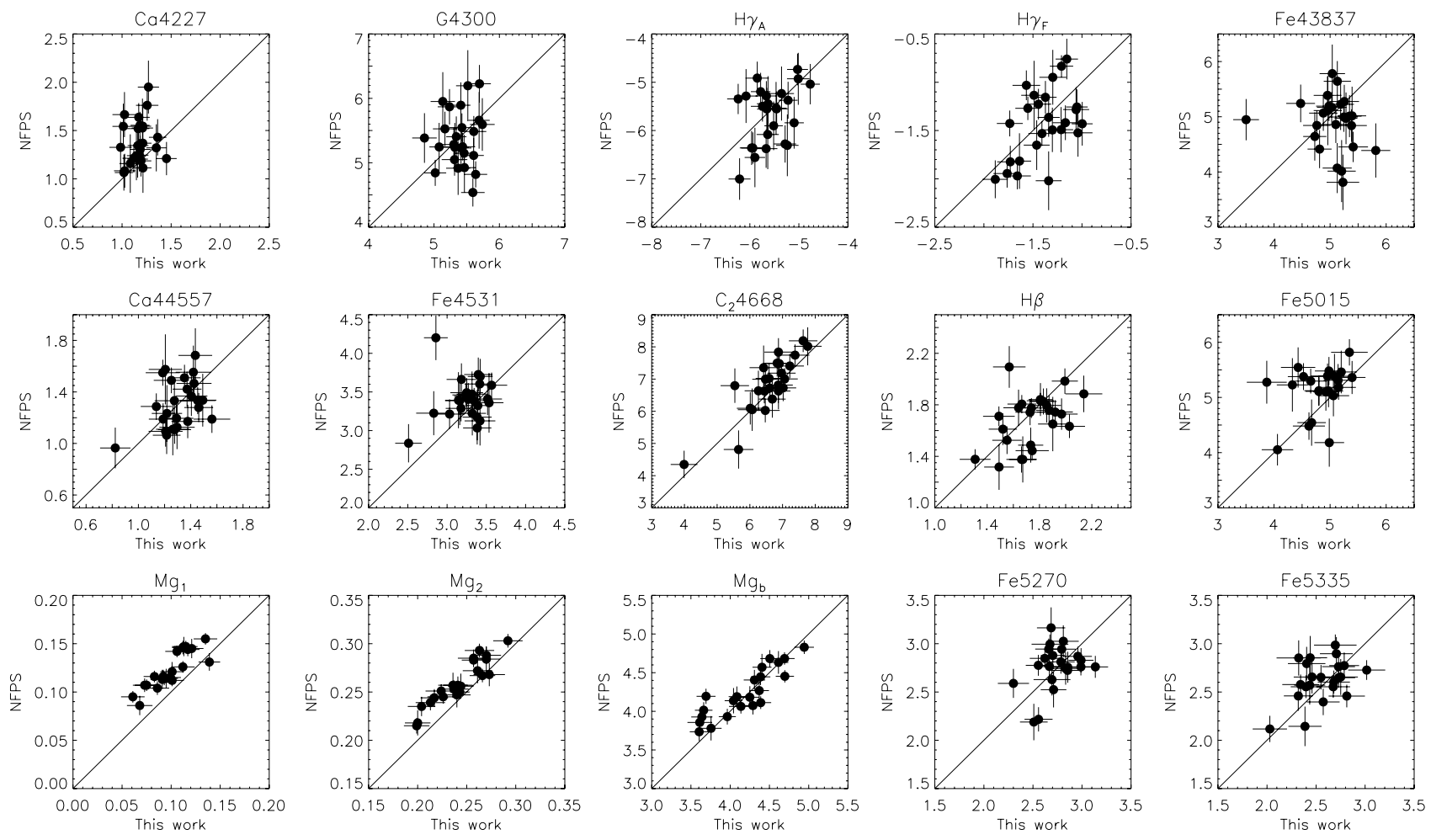

Figure 3. Comparison of our sample with Nelan et al. (2005). The symbols and lines are the same as in Figure 1.

We have demonstrated that our index measurements are in good agreement with those of NFPS, which is one of the highest quality of data available in the Coma cluster so far. Considering our internal and external error analysis, we conclude that our data set has a similar enough quality and our error measurements are well estimated. In the following sections, we only include the NFPS data set for comparison purposes, since this sample also includes velocity dispersions. The NFPS spectra were not 
Table 3

Comparison with Other Studies

\begin{tabular}{|c|c|c|c|c|}
\hline Index & References & $\langle\Delta I\rangle$ & Std & $\sigma_{\exp }$ \\
\hline \multirow[t]{3}{*}{$\mathrm{Ca} 4227$} & $P 01$ & $-0.111 \pm 0.129$ & 0.755 & 0.341 \\
\hline & N05 & $-0.207 \pm 0.047$ & 0.236 & 0.228 \\
\hline & S08 & $0.098 \pm 0.095$ & 0.426 & 0.150 \\
\hline \multirow[t]{3}{*}{ G4300 } & $P 01$ & $-0.217 \pm 0.264$ & 1.519 & 0.567 \\
\hline & N05 & $0.020 \pm 0.094$ & 0.471 & 0.368 \\
\hline & $S 08$ & $-0.146 \pm 0.234$ & 1.046 & 0.328 \\
\hline \multirow[t]{3}{*}{$\mathrm{H} \gamma_{A}$} & $P 01$ & $0.212 \pm 0.304$ & 1.745 & 0.626 \\
\hline & N05 & $0.105 \pm 0.114$ & 0.569 & 0.508 \\
\hline & $S 08$ & $0.359 \pm 0.225$ & 1.004 & 0.368 \\
\hline \multirow[t]{3}{*}{$\mathrm{H} \gamma_{F}$} & $P 01$ & $-0.040 \pm 0.162$ & 0.945 & 0.397 \\
\hline & N05 & $0.037 \pm 0.064$ & 0.320 & 0.242 \\
\hline & $S 08$ & $0.146 \pm 0.146$ & 0.653 & 0.207 \\
\hline \multirow[t]{3}{*}{$\mathrm{Fe} 4383$} & $P 01$ & $-0.970 \pm 0.275$ & 1.604 & 0.789 \\
\hline & N05 & $0.154 \pm 0.139$ & 0.697 & 0.466 \\
\hline & $S 08$ & $-0.113 \pm 0.205$ & 0.916 & 0.420 \\
\hline \multirow[t]{3}{*}{$\mathrm{Ca} 4455$} & $P 01$ & $-0.259 \pm 0.116$ & 0.677 & 0.395 \\
\hline & N05 & $-0.001 \pm 0.038$ & 0.189 & 0.194 \\
\hline & $S 08$ & $-0.089 \pm 0.122$ & 0.547 & 0.194 \\
\hline \multirow[t]{3}{*}{$\mathrm{Fe} 4531$} & $P 01$ & $-0.193 \pm 0.153$ & 0.890 & 0.573 \\
\hline & N05 & $-0.152 \pm 0.066$ & 0.328 & 0.303 \\
\hline & $S 08$ & $-0.206 \pm 0.149$ & 0.668 & 0.318 \\
\hline \multirow[t]{3}{*}{$\mathrm{C}_{2} 4668$} & $P 01$ & $-0.236 \pm 0.345$ & 2.010 & 0.928 \\
\hline & N05 & $0.154 \pm 0.139$ & 0.697 & 0.626 \\
\hline & $S 08$ & $0.140 \pm 0.232$ & 1.037 & 0.650 \\
\hline \multirow[t]{3}{*}{$\mathrm{H} \beta$} & $P 01$ & $0.129 \pm 0.125$ & 0.729 & 0.349 \\
\hline & N05 & $0.071 \pm 0.042$ & 0.210 & 0.163 \\
\hline & $S 08$ & $-0.118 \pm 0.095$ & 0.424 & 0.205 \\
\hline \multirow[t]{3}{*}{ Fe5015 } & $P 01$ & $-0.652 \pm 0.208$ & 1.215 & 0.717 \\
\hline & N05 & $-0.313 \pm 0.102$ & 0.477 & 0.421 \\
\hline & $S 08$ & $-0.243 \pm 0.246$ & 1.098 & 0.805 \\
\hline \multirow[t]{3}{*}{$\mathrm{Mg}_{1}$} & $P 01$ & $-0.012 \pm 0.006$ & 0.037 & 0.014 \\
\hline & N05 & $-0.023 \pm 0.002$ & 0.010 & 0.014 \\
\hline & $S 08$ & $-0.014 \pm 0.003$ & 0.014 & 0.013 \\
\hline \multirow[t]{3}{*}{$\mathrm{Mg}_{2}$} & $P 01$ & $0.004 \pm 0.007$ & 0.041 & 0.017 \\
\hline & N05 & $-0.017 \pm 0.002$ & 0.010 & 0.017 \\
\hline & $S 08$ & $-0.018 \pm 0.004$ & 0.018 & 0.015 \\
\hline \multirow[t]{3}{*}{$\mathrm{Mg}_{b}$} & $P 01$ & $0.015 \pm 0.119$ & 0.692 & 0.339 \\
\hline & N05 & $-0.051 \pm 0.041$ & 0.193 & 0.173 \\
\hline & $S 08$ & $-0.015 \pm 0.115$ & 0.513 & 0.213 \\
\hline \multirow[t]{3}{*}{ Fe5270 } & $P 01$ & $-0.178 \pm 0.112$ & 0.652 & 0.380 \\
\hline & N05 & $-0.018 \pm 0.051$ & 0.241 & 0.196 \\
\hline & $S 08$ & $-0.334 \pm 0.127$ & 0.567 & 0.240 \\
\hline \multirow[t]{3}{*}{ Fe5335 } & $P 01$ & $0.074 \pm 0.098$ & 0.572 & 0.430 \\
\hline & N05 & $-0.065 \pm 0.050$ & 0.233 & 0.226 \\
\hline & $S 08$ & $0.058 \pm 0.146$ & 0.653 & 0.276 \\
\hline
\end{tabular}

Note. Comparison of line strengths measured in this and other studies.

References: Reference in the literature where P01 is from Poggianti et al. (2001a), N05 is from Nelan et al. (2005), and S08 is from Smith et al. (2008); $\langle\Delta I\rangle$ : mean offset between our study and other $\left(I_{\text {here }}-I_{\text {other }}\right)$ and the error in the mean; std: standard deviation of the differences; $\sigma_{\text {exp }}$ : standard deviation expected from the errors.

flux calibrated, and for further analysis we apply an offset to the NFPS data to be consistent with ours according to the average values listed in Table 3.

\section{INDEX-VELOCITY DISPERSION RELATIONS}

In the following paragraphs, we investigate the relationships between different line-strength indices and velocity dispersion $(\sigma)$. We compare these relations between the low- and high- $\sigma$ galaxy samples, and discuss possible parameters that drive the index $-\sigma$ relations (hereafter $\mathrm{I}-\sigma$ ).

\subsection{Results of I- $\sigma$ Relations}

We examine relations between 15 Lick/IDS indices, plus $\langle\mathrm{Fe}\rangle$ and $[\mathrm{MgFe}]^{\prime}$, and the central velocity dispersion of our galaxies in Figure 4. In this plot, we include galaxies from our sample and galaxies in the Coma cluster from NFPS. Objects in common have averaged index values and velocity dispersions. We combined the two data sets and binned index measurements for galaxies of similar velocity dispersion. All the bins have the same narrow interval in $\log \sigma$ of 0.118 dex. The red diamonds represent the average value of each bin weighted by the uncertainties in the index measurements.

Two different types of behaviors emerge between the indices and velocity dispersions for these early-type galaxies in the Coma cluster (Figure 4). One group of indices shows evidence for a break in the slope $\sim 100 \mathrm{~km} \mathrm{~s}^{-1}$, group I, while another group exhibits strong linear relations with $\log \sigma$, group II. We further divide group I into two subgroups where the evidence for a break in the slope is stronger for group Ia than it is for group Ib.

We plot these groups in the different columns of Figure 4. To more easily describe the difference between the groups of I- $\sigma$ trends, the plots also include separate linear fits to low- and high$\sigma$ galaxies (Table 4), even though the correlation coefficients for these fits are low for group I. This figure also contains 13 low- $\sigma$ galaxies, marked as yellow filled circles, which lie outside the model grids and have been excluded from the analysis (discussed in Section 6.1). We refer to these objects as "off-grid" galaxies.

Group I. Galaxies with $\sigma \geqslant 100 \mathrm{~km} \mathrm{~s}^{-1}$ exhibit flat I- $\sigma$ relations in group I. On the other hand, these relations show evidence for a break in their slope for the low- $\sigma$ galaxies. This break is more evident for indices in group Ia than in Ib. Both subgroups show a significantly larger scatter of index values in the low- $\sigma$ regime than for the high- $\sigma$ galaxies. In Table 5, we calculated the standard deviation of the low- and high- $\sigma$ galaxies for group Ia (also shown in Figure 4). The scatter of index values is $\sim$ two times larger for the low $\sigma$ galaxies than it is for the high- $\sigma$ ones. Additionally, the mean value of an index differs for low- and high- $\sigma$ galaxies by $7 \%-27 \%$ for this subgroup. We also performed the Kolmogorov-Smirnov test $(\mathrm{KS})$ to determine whether the two groups of galaxies come from the same distribution. In the same table, we show the KS probability that the two sets are drawn from the same distribution. Since the values of the KS probability are quite small, we conclude that low- and high- $\sigma$ galaxies may indeed come from different populations. We also show the linear fits for this subgroup, although their Spearman-Rank correlation coefficients (see Table 4) are small and these relations are not statistically significant.

The middle panel of Figure 4 shows the Ib subgroup of I- $\sigma$ relations, which also exhibits evidence for the break in the slope around $\sim 100 \mathrm{~km} \mathrm{~s}^{-1}$. However, the break is not as obvious as it is for group Ia since there are a number of low- $\sigma$ galaxies whose index values are equal to or higher than the high- $\sigma$ galaxies. For this reason we perform linear fits for the entire $\sigma$ range together. We take into account both the errors in the index and $\sigma$ and calculate the intrinsic scatter $\left(\sigma_{\text {Intr }}\right)$ and the scatter predicted by the errors $\left(\sigma_{\text {Err }}\right)$ for both low- and high- $\sigma$ galaxies (Table 6$)$. The Spearman-Rank correlation coefficients, $\rho$, are low (lower than 0.5 ) for this subgroup indicating weak or null $\mathrm{I}-\sigma$ correlations, especially for Ca4227. Galaxies with $\sigma<100 \mathrm{~km} \mathrm{~s}^{-1}$ have 

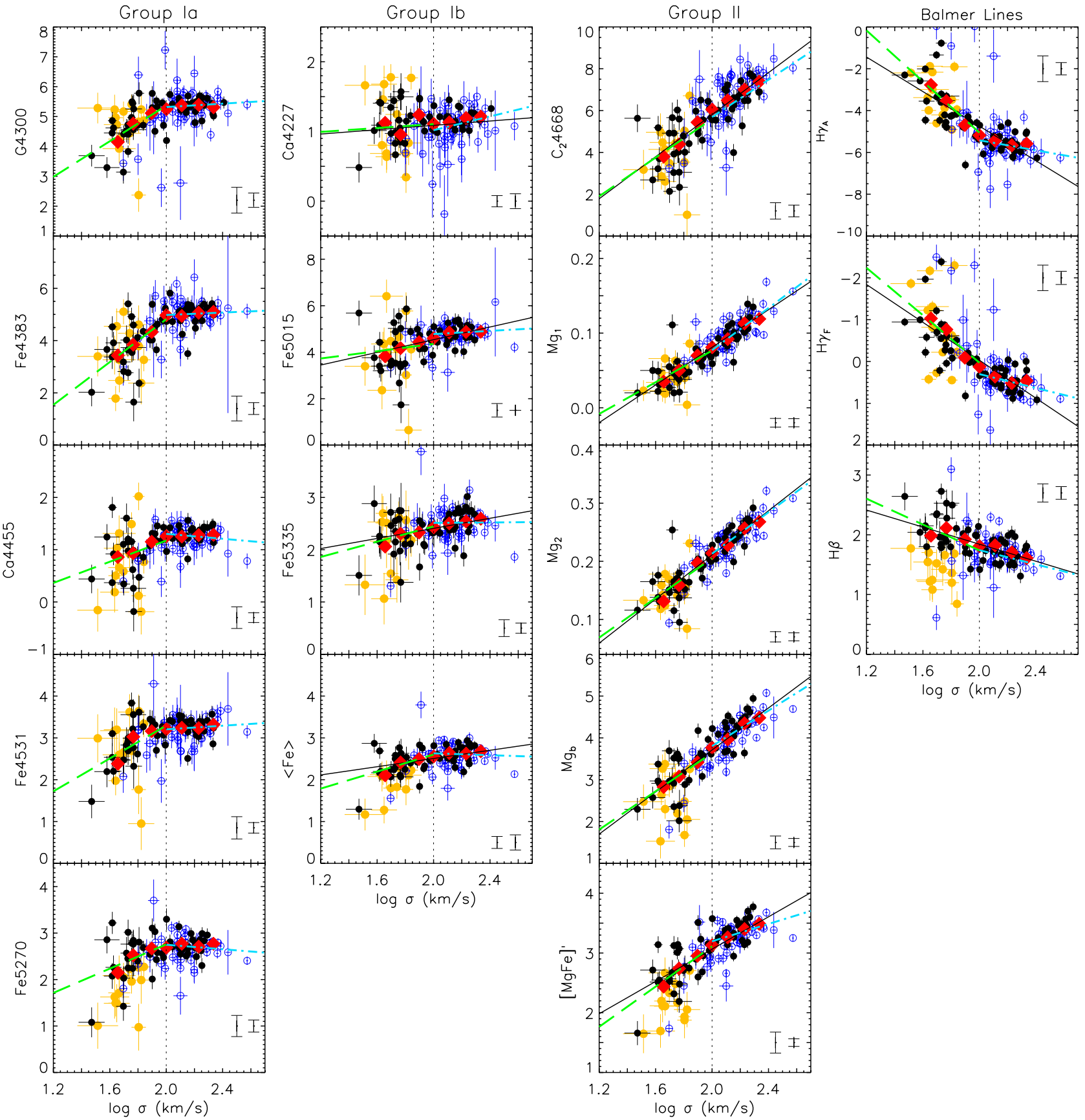

Figure 4. Index- $\log \sigma$ plots. Data from the NFPS sample are marked by the blue open circles, and our sample with black filled circles, and yellow circles for galaxies not fitted by models. We binned the data by $\sigma$ where each bin contains an equal number of objects. The red diamonds are the weighted average values of each bin. The vertical dotted line corresponds to $\sigma=100 \mathrm{~km} \mathrm{~s}^{-1}$. Groups Ia and Ib represent the set of I- $\sigma$ relations that show evidence for a break in the slope, group II I- $\sigma$ with strong linear relations, and the last column contains Balmer lines. In the bottom right corner of I $-\sigma$ plots in Group Ia we show the $1 \sigma$ scatter for low- $\sigma$ on the left and high- $\sigma$ galaxies on the right. In the case of Group Ib and Balmer lines, these lines correspond to the intrinsic scatter for low- and high- $\sigma$, respectively. For Group II, we show the intrinsic and observed scatter, respectively, in the bottom right corner. The black lines represent linear fits for all the galaxies in the individual I- $\sigma$ figures, while the green dashed and light blue dash-dotted lines mark linear fits to low- and high- $\sigma$ galaxies, respectively. Note that the "off-grid" galaxies were not included in the bins and linear regression calculations.

(A color version of this figure is available in the online journal.)

a larger intrinsic scatter ${ }^{4}$ than their more massive counterparts except for $\mathrm{Ca} 4227$ and $\langle\mathrm{Fe}\rangle$ indices (see the lower right-hand corner of Figure 4). However, the larger intrinsic scatter for high- $\sigma$ galaxies in the Ca4227 index is dominated by galaxies from the NFPS data. Ca4227 is the only index for which the

${ }^{4} \sigma_{\mathrm{Intr}}=\sqrt{\frac{\sum_{i=1}^{n}\left(\operatorname{Index}_{i}-\left(\text { intercept }+ \text { slope } \cdot \sigma_{i}\right)\right)^{2}}{N-2}-\frac{\sum_{i=1}^{n} \delta_{\text {Index }_{i}}^{2}+\text { slope }^{2} \cdot \delta_{\sigma_{i}}^{2}}{N}}$ intrinsic scatter is smaller than the scatter due to the errors for both low- and high- $\sigma$ galaxies. The remaining indices in group Ib display a larger intrinsic scatter than the scatter due to errors ( 1.2-2.9 times) for both low- and high- $\sigma$ subsamples, except for Fe5015 for which the intrinsic scatter is zero. The KS test on the residuals between the indices and their respective linear fits implies that only Fe5335 and $\langle\mathrm{Fe}\rangle$ show a high enough probability that the low- and high- $\sigma$ galaxies are drawn from the same population. 
Table 4

I- $\sigma$ Linear Fits

\begin{tabular}{lrrrrrr}
\hline \hline Index & \multicolumn{1}{c}{ Intercept $<100$} & \multicolumn{1}{c}{ Slope $<100$} & \multicolumn{1}{c}{$\rho_{<100}$} & \multicolumn{1}{c}{ Intercept $>100$} & \multicolumn{1}{c}{ Slope $>100$} & $\rho_{>100}$ \\
\hline $\mathrm{Ca} 4227$ & $0.828 \pm 0.640$ & $0.139 \pm 0.352$ & 0.063 & $0.029 \pm 0.590$ & $0.494 \pm 0.268$ & 0.187 \\
$\mathrm{G} 4300$ & $-0.586 \pm 1.717$ & $2.974 \pm 0.944$ & 0.544 & $4.746 \pm 1.140$ & $0.285 \pm 0.519$ & -0.028 \\
$\mathrm{H} \gamma_{A}$ & $7.165 \pm 3.017$ & $-6.110 \pm 1.658$ & -0.652 & $-3.034 \pm 1.852$ & $-1.194 \pm 0.843$ & -0.170 \\
$\mathrm{H} \gamma_{F}$ & $4.644 \pm 1.620$ & $-2.836 \pm 0.890$ & -0.612 & $0.369 \pm 0.834$ & $-0.834 \pm 0.380$ & -0.290 \\
$\mathrm{Fe} 4383$ & $-3.367 \pm 1.731$ & $4.093 \pm 0.951$ & 0.594 & $4.511 \pm 1.091$ & $0.234 \pm 0.497$ & 0.043 \\
$\mathrm{Ca} 4455$ & $-0.854 \pm 0.873$ & $1.015 \pm 0.480$ & 0.371 & $1.773 \pm 0.445$ & $-0.235 \pm 0.203$ & -0.087 \\
$\mathrm{Fe} 4531$ & $-0.629 \pm 1.040$ & $1.962 \pm 0.572$ & 0.426 & $2.773 \pm 0.606$ & $0.216 \pm 0.276$ & 0.016 \\
$\mathrm{C}_{2} 4668$ & $-3.648 \pm 2.460$ & $4.623 \pm 1.352$ & 0.491 & $-3.203 \pm 2.079$ & $4.450 \pm 0.947$ & 0.547 \\
$\mathrm{H} \beta$ & $3.885 \pm 0.945$ & $-1.070 \pm 0.519$ & -0.363 & $2.939 \pm 0.510$ & $-0.596 \pm 0.233$ & -0.254 \\
$\mathrm{Fe} 5015$ & $2.792 \pm 1.920$ & $0.782 \pm 1.055$ & 0.163 & $4.102 \pm 1.027$ & $0.340 \pm 0.469$ & 0.032 \\
$\mathrm{Mg} 1$ & $-0.135 \pm 0.040$ & $0.106 \pm 0.022$ & 0.684 & $-0.204 \pm 0.036$ & $0.141 \pm 0.017$ & 0.702 \\
$\mathrm{Mg}{ }_{2}$ & $-0.136 \pm 0.063$ & $0.171 \pm 0.035$ & 0.652 & $-0.143 \pm 0.045$ & $0.178 \pm 0.020$ & 0.717 \\
$\mathrm{Mg} b$ & $-0.943 \pm 0.981$ & $2.292 \pm 0.539$ & 0.532 & $-0.436 \pm 0.743$ & $2.120 \pm 0.340$ & 0.667 \\
$\mathrm{Fe} 5270$ & $0.151 \pm 0.945$ & $1.303 \pm 0.519$ & 0.257 & $3.236 \pm 0.650$ & $-0.243 \pm 0.297$ & -0.062 \\
$\mathrm{Fe} 5335$ & $0.985 \pm 0.952$ & $0.730 \pm 0.523$ & 0.103 & $2.472 \pm 0.640$ & $0.021 \pm 0.292$ & 0.156 \\
$\langle\mathrm{Fe}\rangle$ & $0.568 \pm 0.813$ & $1.017 \pm 0.447$ & 0.254 & $2.854 \pm 0.586$ & $-0.111 \pm 0.268$ & -0.003 \\
{$[\mathrm{MgFe}]$} & $-0.255 \pm 0.708$ & $1.683 \pm 0.389$ & 0.513 & $1.743 \pm 0.536$ & $0.726 \pm 0.245$ & 0.387 \\
\hline
\end{tabular}

Notes. I- $\sigma$ linear fits for low- and high- $\sigma$ early-type galaxies. The intercept and slope of the I- $\sigma$ linear fit and the Spearman-Rank correlation coefficient $(\rho)$ are presented for low- and high- $\sigma$ galaxies, respectively. Note that the correlation coefficients are low for galaxies in group I implying that for these indices the I $-\sigma$ relations are not significant.

Table 5

I- $\sigma$ Statistics for Group Ia

\begin{tabular}{lccccccc}
\hline \hline Index & $N_{<100}$ & $N_{\geqslant 100}$ & $\bar{I}_{<100}$ & $\bar{I}_{\geqslant 100}$ & std $_{<100}$ & std $\geqslant 100$ & prob $_{\mathrm{KS}}$ \\
\hline $\mathrm{G} 4300$ & 41 & 70 & 4.813 & 5.372 & 0.861 & 0.482 & $3.75 E-06$ \\
$\mathrm{Fe} 4383$ & 41 & 69 & 4.063 & 5.024 & 0.950 & 0.453 & $1.15 E-09$ \\
$\mathrm{Ca} 4455$ & 41 & 69 & 0.988 & 1.258 & 0.413 & 0.186 & $5.82 E-04$ \\
$\mathrm{Fe} 4531$ & 41 & 69 & 2.932 & 3.246 & 0.532 & 0.251 & $2.48 E-04$ \\
Fe5270 & 41 & 66 & 2.516 & 2.704 & 0.456 & 0.260 & $8.01 E-04$ \\
\hline
\end{tabular}

Notes. Statistics for group Ia of I- $\sigma$ plots. $N$ : number of galaxies with $\sigma<100$ $\mathrm{km} \mathrm{s}^{-1}$ and with $\sigma \geqslant 100 \mathrm{~km} \mathrm{~s}^{-1}$ excluding the "off-grid galaxies;" $\bar{I}$ : average value of the index for low- and high- $\sigma$ galaxies; $\operatorname{std}_{<100}$ and std $\geqslant 100$ : standard deviation of the points for the low- and high- $\sigma$ galaxies; prob $\mathrm{KS}$ : the KS probability that the two sets are drawn from the same distribution.

Group II. In the third column of Figure 4 we show the metallic indices which display a tight relation with $\sigma$. This is quantified in Table 7, where we calculated the Spearman-Rank correlation coefficients, and we include the slopes and intercepts of linear fits to these relations. We show the intrinsic scatter and the scatter expected by the errors in the bottom right-hand corner of Figure 4 for each of these indices. For all indices in this group the intrinsic scatter is larger than the scatter due to errors, although the two are very close in value for $\mathrm{Mg}_{1}$ and $\mathrm{Mg}_{2}$. Moreover, the correlation coefficients for all the indices in this group imply a robust relation with $\sigma$. Many studies find that the early-type galaxies in clusters show a tight relation between line-strength index $\mathrm{Mg}_{2}$ and their central velocity dispersion and find similar slopes for this relation. Our slope is consistent with that of NFPS within 1 standard deviation (Table 7).

Balmer Lines. The Balmer lines $\mathrm{H} \gamma_{A}, \mathrm{H} \gamma_{F}$, and $\mathrm{H} \beta$ exhibit negative slopes in their I $\sigma$ relations. Their Spearman-Rank correlation coefficients imply a strong relation for $\mathrm{H} \gamma_{F}$, while the relations for $\mathrm{H} \gamma_{A}$ and $\mathrm{H} \beta$ have slightly lower coefficients, $\rho=-0.665$ and -0.502 , respectively (Table 7). In the case of $\mathrm{H} \beta$, we see a hint for an asymmetric scatter for low- $\sigma$ galaxies where more galaxies have a lower $\mathrm{H} \beta$ index. Together, ours and the NFPS sample have a standard deviation from the linear fit of 0.306 with 23 galaxies having a lower value of their $\mathrm{H} \beta$ index, compared with the scatter of 0.243 for 18 galaxies with a higher $\mathrm{H} \beta$ index. The calculations exclude the galaxies which lie off the model grids.

\subsection{Discussion of Index $-\sigma$ Relations}

Historically, most studies have concentrated on relations between $\mathrm{Mg}_{2}$, a metallicity indicator, and $\mathrm{H} \beta$, an age indicator, with $\log \sigma$, a measure of mass (to name a few: Terlevich et al. 1981; Dressler 1984; Guzman et al. 1992; Bender et al. 1993; Jorgensen et al. 1996; Kuntschner et al. 2001). Recent studies, however, show that these relations are more complex than originally thought. For instance, $\mathrm{Mg}_{2}-\sigma$ may be significantly dependent on both age $(\sim 15 \%)$ and relative abundances of heavy elements $(\sim 20 \%-30 \%)$ (Mehlert et al. 2003; Thomas et al. 2005). While $\mathrm{H} \beta-\sigma$ mainly depends on age, it also changes with metallicity, and chemical composition (Sánchez-Blázquez et al. 2006a).

In this study we present, for the first time, the $\mathrm{Mg}_{2}-\sigma, \mathrm{H} \beta-$ $\sigma$, and relations between other Lick indices with $\sigma$ down to $30 \mathrm{~km} \mathrm{~s}^{-1}$ for a homogeneous sample of early-type galaxies. Our galaxies reside in one of the densest environments in the nearby universe: the center of the Coma cluster. We show that the $\mathrm{Mg}_{2}-\sigma$ relation spans the entire range of $30-260 \mathrm{~km} \mathrm{~s}^{-1}$ with a small scatter. We also investigate relations between the $\mathrm{H} \beta, \mathrm{H} \gamma$ and the metallicity sensitive line strengths with $\sigma$. We discuss the $\mathrm{H} \beta-\sigma$ and $\mathrm{Mg}_{2}-\sigma$ individually, while we examine the remaining indices together.

\subsection{1. $H \beta-\sigma$}

Most studies find that the line-strength $\mathrm{H} \beta$ and the central velocity dispersion of early-type galaxies are anticorrelated. This relation seems to hold in different environments (e.g., Fisher et al. 1995; Jorgensen 1997; Trager et al. 1998; Kuntschner 2000; Caldwell et al. 2003; Nelan et al. 2005; Sánchez-Blázquez et al. 2006a) and is usually interpreted as an interplay between age and mass. However, recent evidence that the $\mathrm{H} \beta-\sigma$ relation is weak or flat in the Coma cluster (Mehlert et al. 2003; SánchezBlázquez et al. 2006a) may allude toward its dependence on the environment. Furthermore, there is some evidence that the age 
Table 6

I- $\sigma$ Linear Fits and Statistics for Index Group Ib

\begin{tabular}{lccccccccc}
\hline \hline Index & $\mathrm{N}$ & Intercept & Slope & $\rho$ & $\sigma_{\text {Int }_{<100}}$ & $\sigma_{\text {Err }_{<100}}$ & $\sigma_{\text {Int }_{2100}}$ & $\sigma_{\text {Err }_{2100}}$ & prob $_{K S}$ \\
\hline C4227 & 111 & $0.614 \pm 0.099$ & $0.262 \pm 0.049$ & 0.159 & 0.197 & 0.217 & 0.242 & 0.256 & 0.200 \\
Fe5015 & 107 & $1.940 \pm 0.282$ & $1.313 \pm 0.136$ & 0.355 & 0.624 & 0.291 & 0.000 & 0.428 & 0.000 \\
Fe5335 & 107 & $1.523 \pm 0.158$ & $0.449 \pm 0.074$ & 0.331 & 0.297 & 0.162 & 0.199 & 0.169 & 0.531 \\
$\langle\mathrm{Fe}\rangle$ & 107 & $1.508 \pm 0.104$ & $0.506 \pm 0.048$ & 0.329 & 0.283 & 0.099 & 0.374 & 0.147 & 0.913 \\
\hline
\end{tabular}

Notes. Statistics for group Ib of I- $\sigma$ plots. $N$ : number of galaxies; Intercept and Slope: linear regression applied to all the galaxies; $\rho$ : Spearman-Rank correlation coefficient; $\sigma_{\text {Int }}$ : intrinsic scatter for the two subsamples, i.e., standard deviation of residuals between the points and the linear fit for each subsample of galaxies (low- and high- $\sigma$ ); $\sigma_{\text {Err }}$ : scatter due to the errors; prob Ks : the KS probability that the two sets are drawn from the same distribution.

Table 7

I- $\sigma$ Linear Fits and Statistics for Index Group II and Balmer Lines

\begin{tabular}{lcrrrrc}
\hline \hline Index & $N$ & \multicolumn{1}{c}{ Intercept } & \multicolumn{1}{c}{ Slope } & \multicolumn{1}{c}{$\sigma_{\text {Int }}$} & $\sigma_{\text {Err }}$ & $\rho$ \\
\hline $\mathrm{C}_{2} 4668$ & 110 & $-3.890 \pm 0.415$ & $4.782 \pm 0.197$ & 0.750 & 0.972 & 0.524 \\
$\mathrm{Mg}_{1}$ & 107 & $-0.177 \pm 0.010$ & $0.128 \pm 0.004$ & 0.844 & 0.016 & 0.011 \\
$\mathrm{Mg}_{2}$ & 107 & $-0.186 \pm 0.012$ & $0.197 \pm 0.006$ & 0.871 & 0.023 & 0.014 \\
$\mathrm{Mg} b$ & 107 & $-1.157 \pm 0.134$ & $2.435 \pm 0.062$ & 0.854 & 0.359 & 0.167 \\
{$[\mathrm{MgFe}]^{\prime}$} & 107 & $0.508 \pm 0.081$ & $1.290 \pm 0.040$ & 0.729 & 0.372 & 0.112 \\
$\mathrm{H} \gamma_{A}$ & 111 & $3.503 \pm 0.229$ & $-4.160 \pm 0.109$ & -0.665 & 1.357 & 0.565 \\
$\mathrm{H} \gamma_{F}$ & 111 & $3.563 \pm 0.126$ & $-2.286 \pm 0.059$ & -0.709 & 0.684 & 0.297 \\
$\mathrm{H} \beta$ & 110 & $3.706 \pm 0.100$ & $-0.943 \pm 0.047$ & -0.502 & 0.380 & 0.190 \\
\hline
\end{tabular}

Notes. Statistics for group II of I- $\sigma$ plots and Balmer lines. $N$ : number of galaxies; intercept and slope of the linear fit take into account both the uncertainties in the index and velocity dispersion; $\rho$ : Spearman-Rank correlation coefficient; $\sigma_{\text {Int }}$ : intrinsic scatter; $\sigma_{\mathrm{Err}}$ : scatter due to the errors.

variation of galaxies in clusters is not large and the $\mathrm{H} \beta-\sigma$ relation may mostly be driven by metallicity Kuntschner \& Davies (in Fornax 1998), or by both variations in global metallicity and relative abundance of different heavy elements for the galaxies in the Coma cluster (Sánchez-Blázquez et al. 2006a).

We confirm that the early-type galaxies in the core of the Coma cluster show a weak anticorrelation between their $\mathrm{H} \beta$ line strength and velocity dispersions. Furthermore, we find a hint toward an asymmetric scatter for the low- $\sigma$ galaxies where more galaxies have higher Balmer line strengths (see last part of Section 5). Similarly, Caldwell et al. (2003, hereafter CRC03) also find a large asymmetric scatter for the low- $\sigma$ galaxies, although in the opposite direction. It is possible that this effect is environmental, since the CRC03 sample includes galaxies in lower-density environments (Virgo, the field, and lower-density environments) than ours. In either case, we extend the $\mathrm{H} \beta-\sigma$ relation to low- $\sigma$ galaxies down to $30 \mathrm{~km} \mathrm{~s}^{-1}$ in $\sigma$, or by 0.2 dex when compared with CRC03. We confirm that $\mathrm{H} \beta$ and $\sigma$ are anticorrelated, and we find a hint of an asymmetric scatter in the low- $\sigma$ regime.

$$
\text { 5.2.2. } M g_{2}-\sigma
$$

Perhaps the most studied $\mathrm{I}-\sigma$ relation is the one of magnesium, in particular, the $\mathrm{Mg}_{2}$ index (just to name a few studies: Terlevich et al. 1981; Gorgas et al. 1990; Guzman et al. 1992; Bender et al. 1993; Bernardi et al. 1998; Colless et al. 1999; Jørgensen 1999; Concannon et al. 2000; Kuntschner 2000; Poggianti et al. 2001a; Proctor \& Sansom 2002; Worthey \& Collobert 2003; Mehlert et al. 2003; Sánchez-Blázquez et al. 2006a). The tight relation between $\mathrm{Mg}_{2}$ and $\sigma$ has been interpreted as evidence that all elliptical galaxies have a low dispersion in age (Bender et al. 1993; Bernardi et al. 1998). Furthermore, the parameter driving this relation has been under much debate. Originally, studies argued that the $\mathrm{Mg}_{2}-\sigma$ relation depended mostly on metallicity (Forbes et al. 1998; Terlevich et al. 1999), while age and relative abundances of different heavy elements have recently been proposed to also influence this relation (Trager et al. 1998; Jørgensen 1999; Trager et al. 2000a; Kuntschner et al. 2001; Poggianti et al. 2001b; Mehlert et al. 2003; Caldwell et al. 2003; Thomas et al. 2005; SánchezBlázquez et al. 2006a).

Our $\mathrm{Mg}_{2}-\sigma$ relation is consistent with other studies in both the slope (Table 7) and the low intrinsic scatter. Although, we note a slightly larger dispersion around the line for low- $\sigma$ galaxies, for the first time, we confirm that this relation is robust for the entire range (30-250 $\left.\mathrm{km} \mathrm{s}^{-1}\right)$ in $\sigma$.

\subsubsection{Index- $\sigma$ Trends in General}

A surprising result in this paper is the different shapes of I $-\sigma$ relations once we include galaxies with $\sigma<100 \mathrm{~km} \mathrm{~s}^{-1}$. Most studies find linear I- $\sigma$ relations for early-type galaxies. However, these samples are limited to galaxies with $\sigma \gtrsim 50 \mathrm{~km}$ $\mathrm{s}^{-1}$. Although our Coma cluster data are not larger than most other studies, it is unique as our sample contains $\sim 40$ galaxies with $30 \leqslant \sigma<100 \mathrm{~km} \mathrm{~s}^{-1}$ (see Paper I).

We find that for the majority of indices, the low- $\sigma$ galaxies exhibit a larger scatter in the I- $\sigma$ relations than the high- $\sigma$ galaxies. Concannon et al. (2000) also found that the scatter in index $-\sigma$ relations is larger for low-mass galaxies. Their interpretation of this result for the $\mathrm{H} \beta-\sigma$ relation is that the lowmass galaxies have experienced a more varied star formation history and have a larger spread in age. Similarly, SánchezBlázquez et al. (2006a) show that the scatter in the $\mathrm{I}-\sigma$ relations is mainly a consequence of the element abundances varying with age. We investigated whether the shapes of the I- $\sigma$ relations are related to the variations in individual element abundances using Tripicco \& Bell (1995), Thomas et al. (2003), and Korn et al. (2005).

Indices in the left column (group Ia) of Figure 4 have strong Fe dependence in common, except for Fe4531 and G4300 which mostly depend on $\mathrm{Ti}$ and to a lesser extent on $\mathrm{Fe}$. Group $\mathrm{Ib}$ contains indices which depend on both, $\alpha$-peak elements and Fe. In group II, we again find a mixture of elements driving the indices. Carbon and $\alpha$-peak elements, $\mathrm{Mg}$ and $\mathrm{O}$ in particular, do appear to influence most indices in this column, with the exception of $[\mathrm{MgFe}]^{\prime}$ which does not depend much on the $[\alpha / \mathrm{Fe}]$ ratio (Thomas et al. 2003). Finally, $\mathrm{H} \gamma_{A, F}$ indices depend on the $[\alpha / \mathrm{Fe}]$ ratio although this dependency diminishes with increasing metallicity (Korn et al. 2005), while the $\mathrm{H} \beta$ index is moderately influenced by elemental abundance ratios. If the abundance ratios are what drives the relation between the higher order Balmer lines and $\sigma$, then there is a possibility that the larger scatter of these indices toward the low- $\sigma$ galaxies is caused by 
the decreased metallicity. In conclusion, we do not find any clear correlations between the shape of the I- $\sigma$ relations with the element abundance driving the indices, neither with $\alpha$-nor with Fe-peak elements.

Poggianti et al. (2001a) find that the slopes of the indexmagnitude relations can be explained by trends between the age and metallicity with luminosity. These relations are an alternate form of the I- $\sigma$ relations, since magnitude and $\sigma$ are related via the Faber-Jackson relation. Sánchez-Blázquez et al. (2006a) find that variations in these two parameters are not sufficient to explain the I- $\sigma$ slopes. They conclude that a likely explanation for the different $\mathrm{I}-\sigma$ relations could be the relative abundance of elements $[\mathrm{Mg} / \mathrm{Fe}],[\mathrm{C} / \mathrm{Fe}]$, and $[\mathrm{N} / \mathrm{Fe}]$, as already speculated by other authors (Worthey et al. 1992; Greggio 1997; Jorgensen 1997; Kuntschner 2000; Trager et al. 2000a; Thomas et al. 2002; Mehlert et al. 2003; Thomas et al. 2005). More specifically, Sánchez-Blázquez et al. (2006a) show that the I- $\sigma$ slopes are best reproduced when the $\alpha$-peak elements change more than the Fe-peak elements and, the $[\mathrm{Mg} / \mathrm{Fe}]$ and $[\mathrm{N} / \mathrm{Fe}]$ ratios change more than the rest of the alpha elements with $\sigma$. We find no clear evidence in support of these results.

The answer to the different shapes of $\mathrm{I}-\sigma$ relations may lie in the finding that $\mathrm{C}_{2} 4668, \mathrm{Mg}_{1}, \mathrm{Mg}_{2}$, and $\mathrm{Mg}_{b}$, all in group II (exhibiting robust linear relations), are independent of the microturbulent velocity of stellar atmospheres (Tripicco $\&$ Bell 1995). This is unusual, since most other indices depend on this parameter. In fact, according to Tripicco \& Bell (1995), changing the microturbulent velocity of stellar atmospheres just by $1 \mathrm{~km} \mathrm{~s}^{-1}$ causes changes in the indices which are more significant than if one were to double the metal abundance. Hence, the shape and/or tightness of the $\mathrm{I}-\sigma$ relations for metallic lines may be determined by how much an index depends on the microturbulent velocity of the underlying stellar atmospheres.

\section{AGES, METALLICITIES, AND ELEMENT ABUNDANCES OF EARLY-TYPE GALAXIES}

One of the main goals of this paper is to derive ages and metallicities of faint early-type galaxies in the central region of the Coma cluster. This is possible through evolutionary stellar synthesis models. We use index-index diagrams to compare the observed line strengths with the SPM of Thomas et al. (2004, hereafter TMK04), an extension of TMB03, to derive the ages, metallicities, and abundance ratios for our sample of galaxies.

The models that we use are based on the evolutionary population synthesis code from Maraston (1998). They account for element ratio changes based on the response functions from Korn et al. (2005) via a method similar to the one introduced by Trager et al. (2000b). The TMB03 models span a range in age between 1 and $15 \mathrm{Gyr}$, total metallicity, $[\mathrm{Z} / \mathrm{H}]$, from -2.25 to 0.65 , and the $\alpha$-ratio values of $0.0-0.5$. Using an age-sensitive index versus a metallicity-sensitive index with the models allows for a derivation of ages, metallicities, and $\alpha$-ratios of galaxies.

We use $\mathrm{H} \beta$ as the main indicator of age. This line strength is only marginally sensitive to the $\alpha / F e$ ratio, while the higher order Balmer lines are significantly affected by $[\alpha / \mathrm{Fe}]$ at supersolar metallicities (TMK04). Furthermore, $\mathrm{H} \beta$ is a prominent feature in the spectra of our galaxies. As a metallicity gauge we use the $[\mathrm{MgFe}]^{\prime}$ index as defined by TMB03, albeit it is also dependent on age. This index is sensitive to the overall metallicity and, similarly to $\mathrm{H} \beta$, depends little on the $[\alpha / \mathrm{Fe}]$ ratio.

We use a combination of the $\mathrm{H} \beta-[\mathrm{MgFe}]^{\prime}$ and $\langle\mathrm{Fe}\rangle-\mathrm{Mg}_{b}$ indices to determine the ages, metallicities, and the $\alpha$-ratios for our Coma galaxies. However, before investigating the relations between age, metallicity, and $[\alpha / \mathrm{Fe}]$ we note that, due to the tilt of the model grids and the given errors in individual indices, the errors in derived ages and metallicities are likely to be correlated (Kuntschner et al. 2001; Terlevich \& Forbes 2002). In order to reduce the error in the line-strength indices (and therefore the correlated errors in the derived parameters) we have obtained an average value of each index for galaxies with similar velocity dispersions (for $\sigma$ bins, see Table 8). These average values binned by velocity dispersion represent "average" or "binned" galaxies.

As a precursory step, we plot our galaxies on top of the model grids in Figure 5. In the left panel of this figure, we fixed the $\alpha$-ratio to the solar value according to the findings of Gorgas et al. (1997), so that we can determine the ages and metallicities. While in the $\langle\mathrm{Fe}\rangle-\mathrm{Mg}_{b}$ panel, the age is set to $6 \mathrm{Gyr}$, as this is an average age of our low- $\sigma$ galaxies and corresponds to the ages derived from our Paper I. Once the age is at a fixed value, we can determine the metallicities and the $[\alpha / \mathrm{Fe}]$. We marked the low- and high- $\sigma$ galaxies with different symbols and also included the "average galaxies" for which the indices are binned by velocity dispersion.

The Coma cluster galaxies in our sample exhibit a wide range in both their ages and metallicities. Further, the more massive galaxies have, on average, metallicities equal to or larger than solar, while the low- $\sigma$ galaxies (the three smallest diamonds) have on average, subsolar metallicities and younger ages. Similar results of wide age and metallicity ranges have already been noted by other authors in the literature for both the Coma cluster (Jørgensen 1999; Poggianti et al. 2001a; Mehlert et al. 2003; Nelan et al. 2005; Sánchez-Blázquez et al. 2006b), and for the lower density environments (Caldwell et al. 1993; Jorgensen 1997; Trager et al. 1998, 2000b; Nelan et al. 2005; Sánchez-Blázquez et al. 2006b; Bernardi et al. 2006).

Our sample seems to split around $[\mathrm{MgFe}]^{\prime} \sim 3$, or more precisely around the solar metallicity. This is in agreement with Poggianti et al. (2001a) who find that their faint Coma cluster galaxies are divided into two groups, one being metal rich and the other one metal poor. In our sample, galaxies with the supersolar metallicities are predominantly high- $\sigma$ earlytypes. A group of low- $\sigma$ galaxies is also present in this regime and these galaxies are on average younger than the high$\sigma$ galaxies. In contrast, all the other low- $\sigma$ galaxies exhibit subsolar metallicities. This result may imply two different formation mechanisms for low- $\sigma$ early-type galaxies within the Coma cluster. Alternatively, galaxies entering the cluster environment at different epochs would be stripped from their gas at different evolutionary stages, possibly explaining the metallicity differences. An investigation of these parameters and their dependence on the position within the cluster and the cluster environment is a topic of our future papers.

On the other hand, Mehlert et al. (2003) and Thomas et al. (2005) find that their samples of early-type galaxies split into two subclasses at $\mathrm{H} \beta \sim 2 \AA$ where the younger subclass has solar or higher metallicities on the $\mathrm{H} \beta-[\mathrm{MgFe}]^{\prime}$ plot. Mehlert et al. (2003) find that the "young clump" (their Figure 4) is dominated by S0 galaxies, rather than Es. Thomas et al. (2005) attribute this division to either younger stellar populations or blue horizontal branch stars. Here too, we can argue that such a division exists for the low- $\sigma$ galaxies in our sample, but not for the more massive Es. We denote a group of galaxies with old ages and low metallicities, while the remaining low- $\sigma$ galaxies in our sample have intermediate ages and a large range in metallicity. 

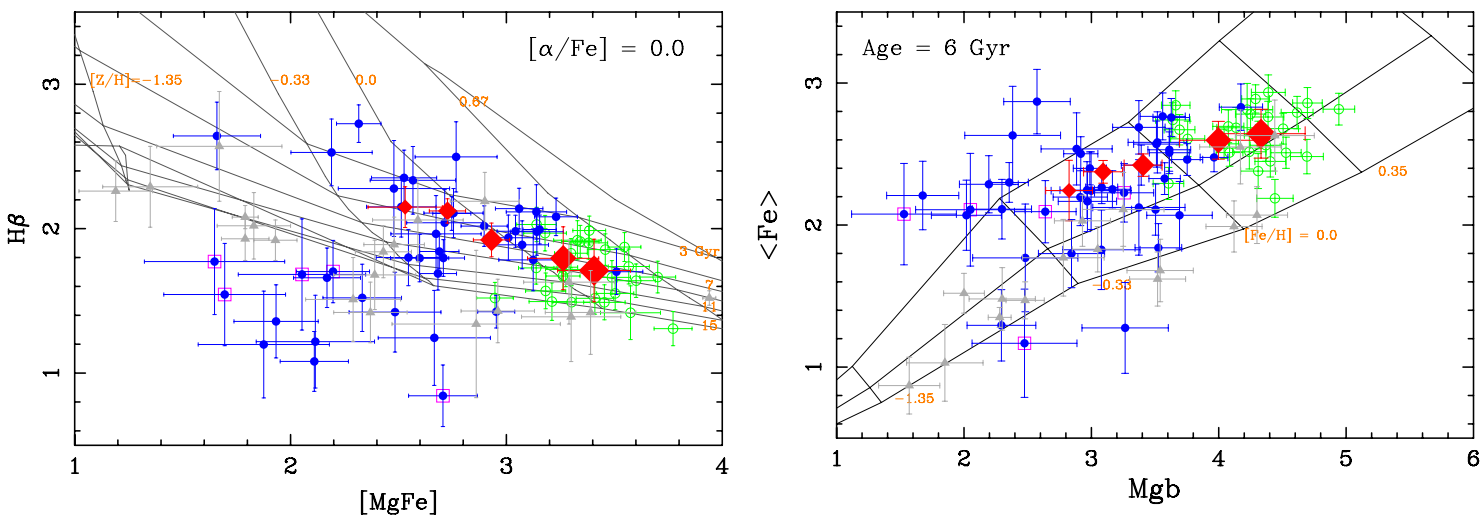

Figure 5. $[\mathrm{MgbFe}]^{\prime}$ vs. $\mathrm{H} \beta$ and $\mathrm{Mgb}$ vs. $\langle\mathrm{Fe}\rangle$ plots for age of $6 \mathrm{Gyr}$ plot. The low- and high- $\sigma$ galaxies are represented by the blue solid and green open circles, respectively. We mark the nucleated dEs (Graham \& Guzmán 2003) with purple open squares. The large red solid diamonds denote the galaxies binned by velocity dispersion where each bin is of an equal interval in $\log \sigma$. The larger diamonds represent the more massive galaxies with larger $\sigma$. The bins do not include galaxies which lie outside the model grids. For comparison, we also include GCs from Cenarro et al. (2007) as the gray triangles.

(A color version of this figure is available in the online journal.)

Table 8

Velocity Dispersions, Ages, Metallicities, and $[\alpha / \mathrm{Fe}]$ for Binned Galaxies

\begin{tabular}{lrccccc}
\hline \hline Bin & $N$ & $\log \sigma$ Range & $\langle\log \sigma\rangle$ & \multicolumn{1}{c}{$\langle\log$ Age $\rangle$} & $\langle[\mathrm{Z} / \mathrm{H}]\rangle$ & $\langle[\alpha / \mathrm{Fe}]\rangle$ \\
\hline 1 & 5 & $2.291<\sigma<2.127$ & $0.893 \pm 0.092$ & $0.150 \pm 0.069$ & $0.255 \pm 0.035$ & $2.212 \pm 0.020$ \\
2 & 12 & $2.127<\sigma<1.963$ & $0.880 \pm 0.064$ & $0.086 \pm 0.027$ & $0.202 \pm 0.023$ & $2.053 \pm 0.016$ \\
3 & 8 & $1.963<\sigma<1.799$ & $0.765 \pm 0.102$ & $-0.041 \pm 0.078$ & $0.179 \pm 0.048$ & $1.890 \pm 0.016$ \\
4 & 13 & $1.799<\sigma<1.635$ & $0.721 \pm 0.066$ & $-0.085 \pm 0.043$ & $0.162 \pm 0.037$ & $1.744 \pm 0.008$ \\
5 & 4 & $1.635<\sigma<1.400$ & $0.778 \pm 0.094$ & $-0.111 \pm 0.147$ & $0.143 \pm 0.031$ & $1.610 \pm 0.010$ \\
\hline
\end{tabular}

This suggests that some low-mass early-type galaxies harbor younger stellar populations, while the others are old and metal poor. We also found no correlation with morphology for this result.

The right panel of Figure 5 shows a relation between the $\mathrm{Mg} b$ and $\langle\mathrm{Fe}\rangle$ indices overlaid with models. When the age is fixed, it is possible to derive the $[\alpha / \mathrm{Fe}]$ ratios for these galaxies. Similar to the $[\mathrm{MgFe}]^{\prime}$ versus $\mathrm{H} \beta$ plot, there is a division in the sample between galaxies around the solar metallicity in this figure. Majority of galaxies with supersolar metallicities are high- $\sigma$ galaxies. They cluster around $[\alpha / \mathrm{Fe}]=0.3$ which is consistent with the well known overabundance of $\mathrm{Mg}$ among Es, i.e., a depression of $\mathrm{Fe}$ with respect to the solar values (Trager et al. 2000a). Although the low- $\sigma$ galaxies show a wider range in $\alpha$-ratios $(0.0-0.5)$ than their more massive counterparts, the majority of low- $\sigma$ galaxies, with the exception of a few objects, have low $[\alpha / \mathrm{Fe}] \lesssim 0.2$. This result indicates that the low-mass galaxies have had a more extended star formation history (Gorgas et al. 1997).

\subsection{Off-Grid Galaxies}

Our sample contains $13 \mathrm{dE} / \mathrm{dS} 0$ galaxies that are not fitted by the models when using $\mathrm{H} \beta-[\mathrm{MgFe}]^{\prime}$ indices (the bottom left corner of Figure 5). To see whether we can recover their ages, we plot $\mathrm{H} \gamma_{A}$ and $\mathrm{H} \gamma_{F}$ with $[\mathrm{MgFe}]^{\prime}$ in Figure 6. Even when we use a different Balmer line strength, $\mathrm{H} \gamma$, eight out of 13 galaxies still lie off the model grids. All of these galaxies are low mass with $30 \leqslant \sigma<70 \mathrm{~km} \mathrm{~s}^{-1}$. In the following paragraphs, we perform a number of tests to determine whether these galaxies truly have such low values of the $\mathrm{H} \beta$ index.

First, we checked for a possibility of nebular emission in the $\mathrm{H} \beta$ feature, as it would make this line strength appear weaker, i.e., yielding older ages. Aside from the test that we have already performed by dividing each spectra with its optimal template (see Section 3), we also stacked the spectra of these galaxies together (since they have a small range in $\sigma$ ) at the original resolution $(\mathrm{FWHM}=1.9 \AA$ ) and checked for any possible emission in the $\mathrm{H} \beta$ absorption feature (Figure 7) which was not detected previously. At this resolution, we do not find any contamination of the off-grid galaxies by nebular emission.

Second, we considered possible sky subtraction and scattered light problems. If scattered light was the cause of the low- $\mathrm{H} \beta$ index values, not only $\mathrm{H} \beta$ but the rest of the indices should be affected as well. Further, wrongly subtracted sky levels should also lead to asymmetrical residuals at the locations of the bright sky lines. However, this was not the case in our spectra, and we exclude sky subtraction and the scattered light correction as causes of the low- $\mathrm{H} \beta$ index values.

Third, we checked whether these off-grid galaxies were consistent with the position of globular clusters (hereafter $\mathrm{GCs})$ on the $[\mathrm{MgFe}]^{\prime}$ versus $\mathrm{H} \beta$ plot. If the models would extend to these galaxies, they would correspond to very old and very metal-poor objects similar to GCs or they could also be "primordial" as suggested by Rakos \& Schombert (2004). Figure 5 shows a possibility that some of the off-grid galaxies are consistent with GCs, while a number of these galaxies lie in a region even older and more metal poor than GC.

We also checked whether the $\mathrm{H} \beta / \mathrm{H} \gamma_{A, F}$ ratios for the off-grid galaxies are consistent with the other galaxies in our sample and with GCs from Cenarro et al. (2007). This is shown in Figure 8. The off-grid galaxies deviate noticeably from the $\mathrm{H} \gamma_{A}$ and $\mathrm{H} \gamma_{F}$ with $\mathrm{H} \beta$ plots when compared with other Coma galaxies in our sample and the GCs. Interestingly, the off-grid galaxies show no deviations in the $[\mathrm{Mg} / \mathrm{Fe}]$ plot. This points toward a possibility 

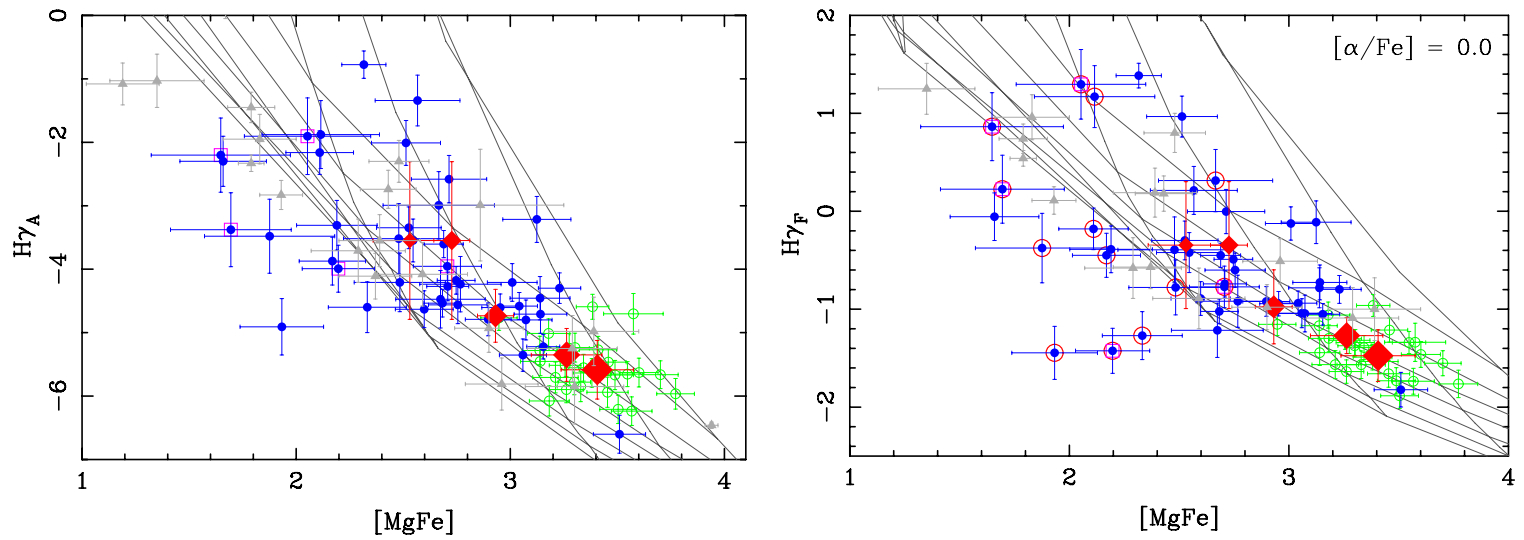

Figure 6. $[\mathrm{MgbFe}]^{\prime}$ vs. $\mathrm{H} \gamma_{A}$ and $\mathrm{H} \gamma_{F}$ plots. The low- and high- $\sigma$ galaxies are represented by the blue solid and green open circles, respectively, and GCs by the gray triangles. We mark galaxies which are not fitted by the models from Figure 5 with red open circles.

(A color version of this figure is available in the online journal.)

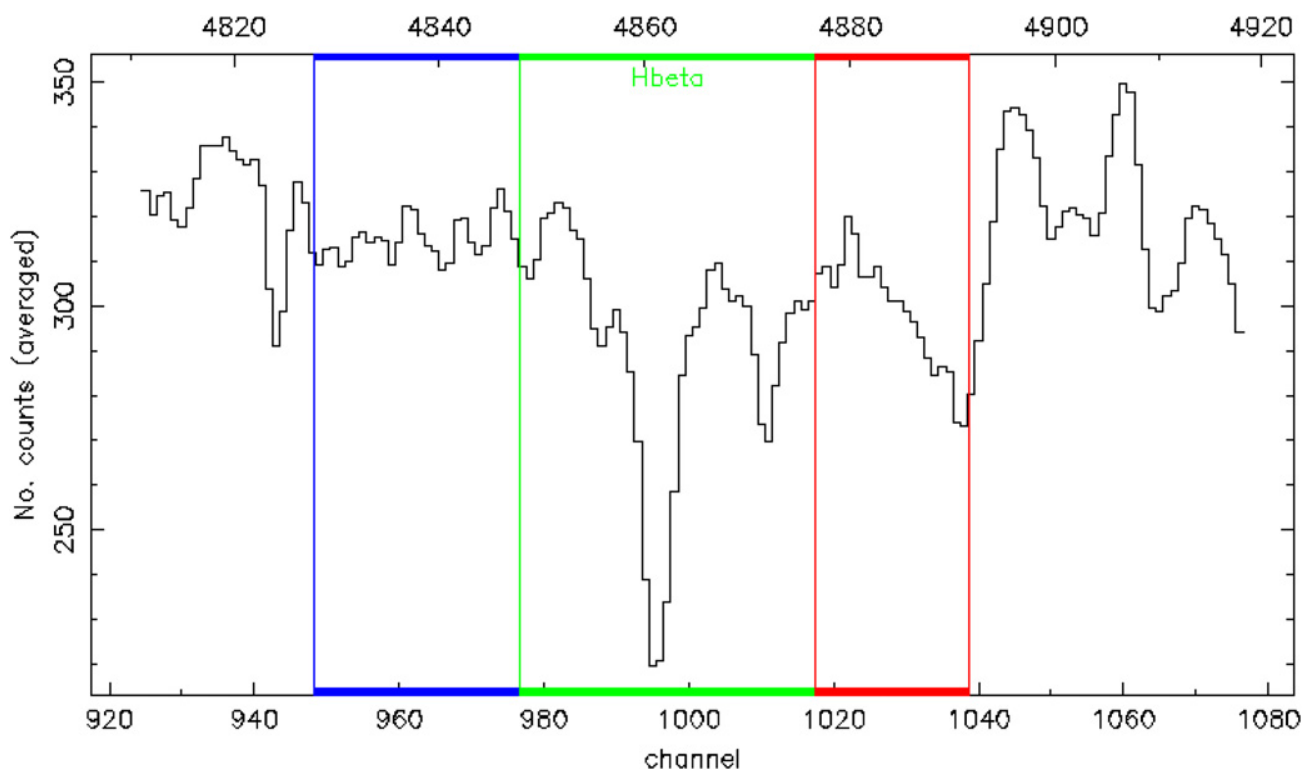

Figure 7. $\mathrm{H} \beta$ of the off-grid galaxies combined. We stacked the spectra of 13 galaxies which did not fit on the models for the [MgFe]' vs. $\mathrm{H} \beta$ plot.

(A color version of this figure is available in the online journal.)

of some problems in the measurements of the Balmer lines for these off-grid galaxies, which is not necessarily true for the other indices.

Finally, we were able to compare our spectra with those of S08 (also R. Smith 2008, private communication). The comparison showed that the spectra of the off-grid galaxies likely suffered from some spurious high-resolution frequency patterns found only in the Balmer continuum bands used to measure the index. However, this is not the case for the rest of our galaxies which have good quality. To err on the safe side we exclude these galaxies from the analysis in this paper.

\subsection{Method of Deriving SPM Parameters}

We use an iterative procedure similar to Thomas et al. (2005) to derive the ages, metallicities, and $\alpha$-ratios for our sample of early-type galaxies in the Coma cluster. This procedure consists of, first, determining the age and metallicity of each galaxy by interpolating the model grids for the $[\mathrm{MgFe}]^{\prime}-\mathrm{H} \beta$ plot, at a given $\alpha$-ratio. This particular combination of indices has low sensitivity to abundance ratios and is therefore well suited for determining the other two SPM parameters, age and metallicity. Then, we fix the age as it was derived in the first step, and we derive the $[\alpha / \mathrm{Fe}]$ and metallicity with $\langle\mathrm{Fe}\rangle-\mathrm{Mg}_{b}$ index combination. This two-step procedure is repeated until the metallicities derived from $[\mathrm{MgFe}]^{\prime}-\mathrm{H} \beta$ and $\langle\mathrm{Fe}\rangle-\mathrm{Mg}_{b}$ match well (i.e., better than $15 \%$ difference).

We used the same iterative procedure for deriving the error ellipse in age, metallicity, and $\alpha$-ratio values for each galaxy. We treated the extremes of the index uncertainties as individual values. Conservatively, we chose the highest value of the error ellipse for the uncertainty in the SPM parameter. The ages, metallicities, and $\alpha$-ratios together with their respective errors are shown in Tables 1 and 2, while these parameters for the binned galaxies are in Table 8 .

Our data of early-type Coma galaxies span a wide range in all SPM parameters. The low- $\sigma$ galaxies have on average: lower ages, $6.1 \pm 0.1$ versus $8.9 \pm 0.1$ Gyr for high- $\sigma$ galaxies; lower metallicities, $-0.050 \pm 0.003$ versus $1.131 \pm 0.004 \mathrm{dex}$; and slightly lower $[\alpha / \mathrm{Fe}], 0.173 \pm 0.002$ versus $0.238 \pm 0.002$, closer to the solar value of 0.0 . Here, we excluded the galaxies which lie off the model grids as discussed in Section 6.1. 

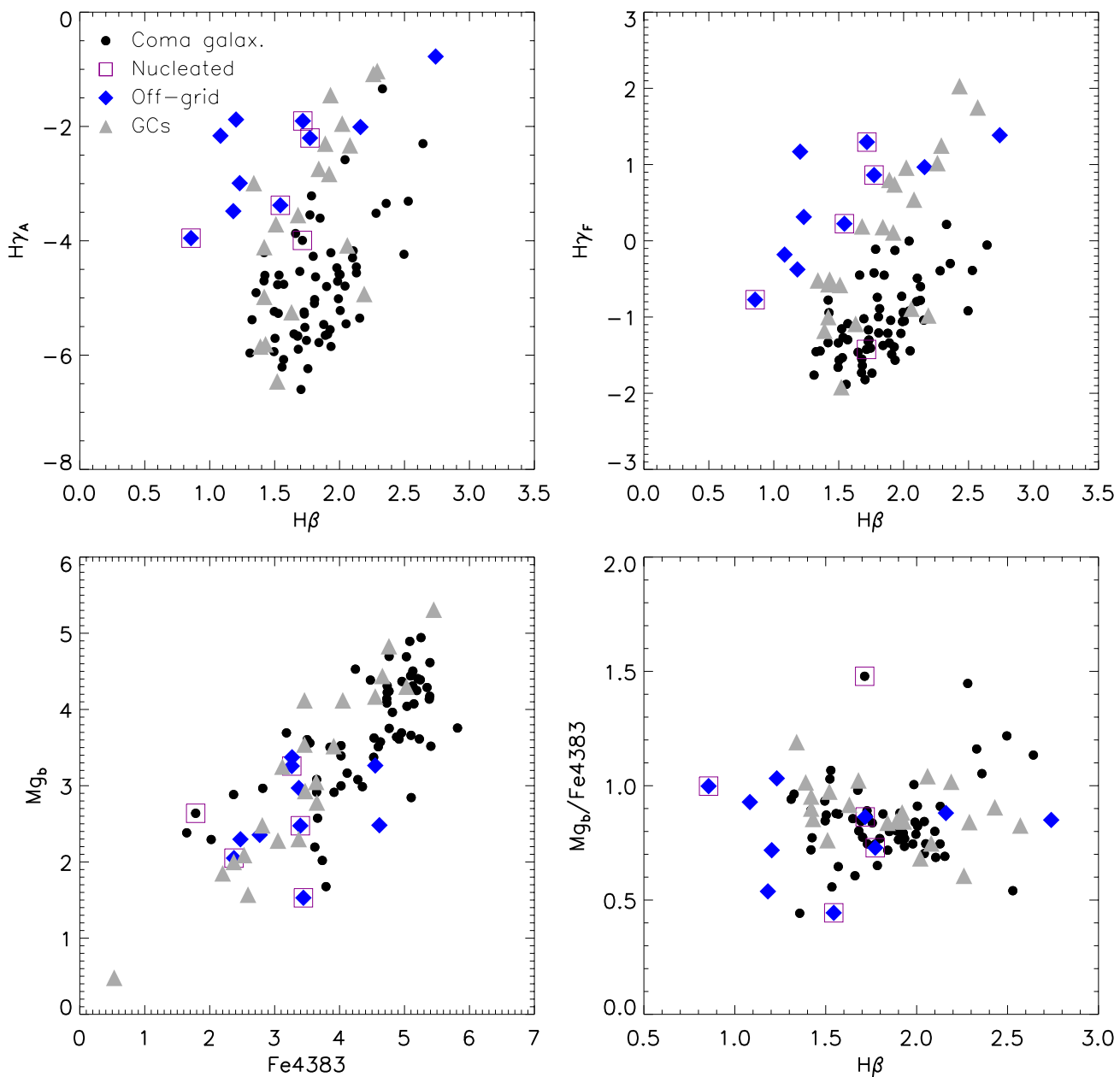

Figure 8. Testing the off-grid galaxies. The top left and right plots show $\mathrm{H} \beta$ index vs. the higher order Balmer lines $\mathrm{H} \gamma_{A}$ and $\mathrm{H} \gamma_{F}$. The bottom plots investigate whether there are any inconsistencies in the $[\mathrm{Mg} / \mathrm{Fe}]$ between the off-grid galaxies with the other galaxies in the sample and GCs (Cenarro et al. 2007). The black filled circles represent the Coma galaxies from our sample where the blue diamonds are off-grid galaxies. Nucleated dEs are marked by purple open squares and the GCs by gray triangles.

(A color version of this figure is available in the online journal.)

\section{MODEL PARAMETERS VERSUS $\sigma$}

In the index-index figures overlaid with models and mentioned in the previous section, we note a trend with mass for our galaxies. Henceforth, we plot the model parameters, age, metallicity, and $[\alpha / \mathrm{Fe}]$ versus $\log \sigma$ for the five velocity dispersion bins (Figure 9). We also include the individual galaxies in these plots although we perform the linear least-squares regression (see Table 9) for the binned data only. The bin values for the age, metallicity, and $[\alpha / \mathrm{Fe}]$ were calculated by averaging these parameters for individual galaxies within each bin. The errors in the model-derived average parameters were determined by taking the standard deviation of the individual parameter values within each bin and then dividing by the square root of the number of galaxies in the bin. Although not statistically significant, trends emerge between the age, metallicity, and $[\alpha / \mathrm{Fe}]$ with $\sigma$, and we compare them to the same relations from Nelan et al. (2005)..$^{5}$

\footnotetext{
5 We excluded one galaxy from the linear regression (GMP 2585) since this galaxy is clearly an outlier in both metallicity- and $[\alpha / \mathrm{Fe}]-\sigma$ plots and it has an unusually low metallicity and a high value of $[\alpha / \mathrm{Fe}]$.
}

\subsection{Age $-\sigma$}

The top panel of Figure 9 shows the relation between $\log$ age and $\log \sigma$. The linear fit between these two parameters is uncertain due to the large errors in age. However, both the binned and the individual galaxies in this figure provide clear evidence for a trend between age and $\sigma$ where the low- $\sigma$ galaxies display younger ages.

Within the errors, our age- $\log \sigma$ trend is consistent with Nelan et al. (2005), although there are some differences. Nelan et al. (2005) find that the age- $\log \sigma$ relation steepens for the low-mass galaxies. We do not find this effect, since the age- $\log \sigma$ for these galaxies levels off at $\sim 4 \mathrm{Gyr}$ in our case.

Whether the age $-\sigma$ relation exists for early-type galaxies or not is still an unresolved issue in the literature. Jørgensen (1999), Kuntschner et al. (2001), Mehlert et al. (2003), Thomas et al. (2005), and Sánchez-Blázquez et al. (2006b) do not find a relation between these parameters, although results from Sánchez-Blázquez et al. (2006b) yield a relation for galaxies in low-density environments. However, at least a trend between age and $\sigma$ is found in the samples of Concannon et al. (2000), Poggianti et al. (2001a), Caldwell et al. (2003), Proctor et al. (2004), Nelan et al. (2005), and Bernardi et al. (2006). Additionally, Nelan et al. (2005) derive age $-\sigma$ relations for 


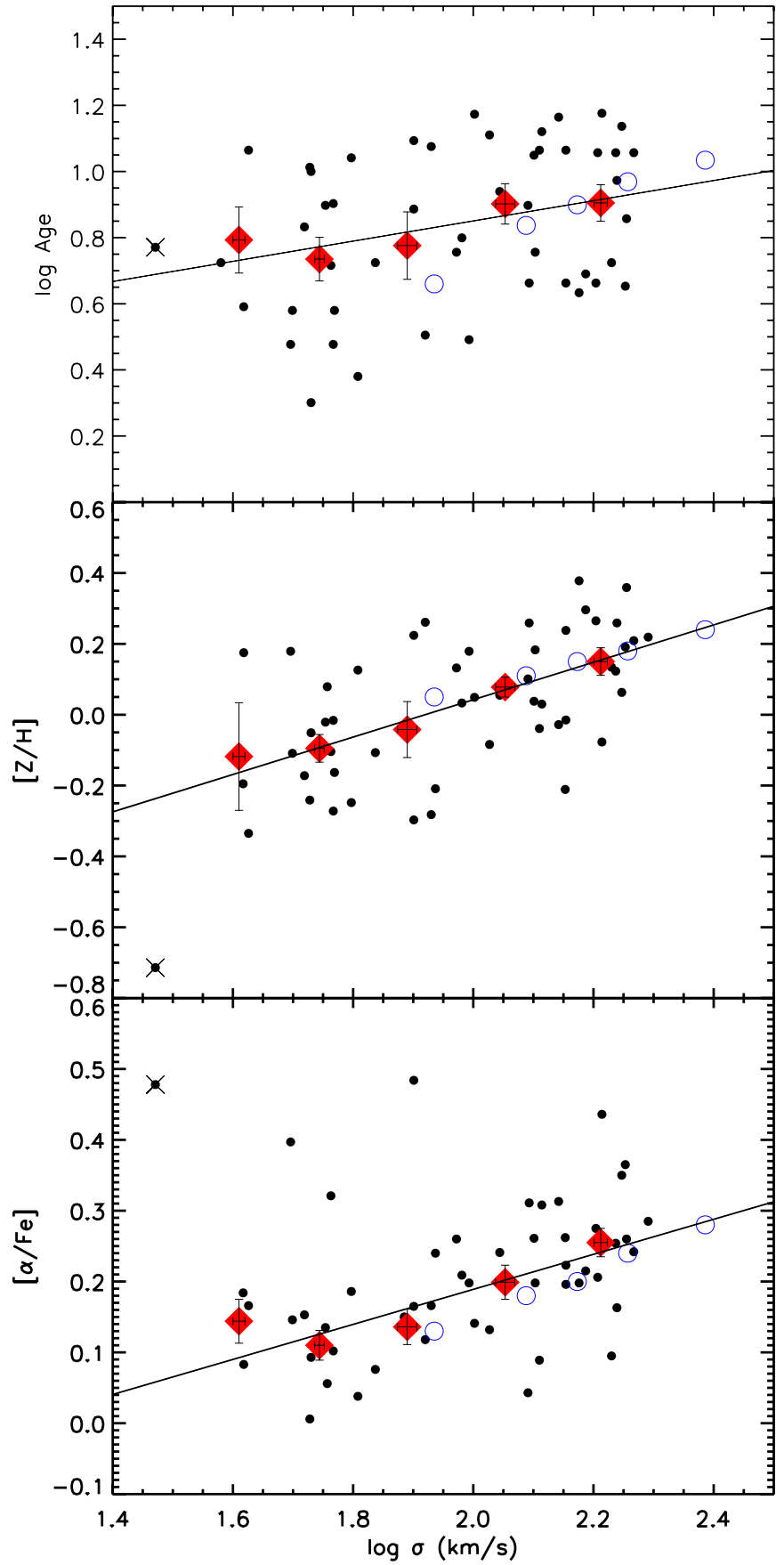

Figure 9. $\log \sigma$ vs. age, metallicity, and $[\alpha / \mathrm{Fe}]$ (from top to bottom). The small filled circles are individual galaxies in our data set, the red diamonds represent "average" galaxies whose indices were binned by velocity dispersion prior to deriving the SPM parameters, and the blue open circles represent the NFPS data without any offsets applied.

(A color version of this figure is available in the online journal.)

Table 9

Model Parameters Versus $\sigma$ Relations

\begin{tabular}{lcc}
\hline \hline$\sigma$ Relation & Intercept & Slope \\
\hline $\log ($ age $)$ vs. $\sigma$ & $0.22 \pm 0.34$ & $0.31 \pm 0.18$ \\
{$[\mathrm{Z} / \mathrm{H}]$ vs. $\sigma$} & $-1.00 \pm 0.26$ & $0.53 \pm 0.13$ \\
{$[\alpha / \mathrm{H}]$ vs. $\sigma$} & $-0.13 \pm 0.13$ & $0.17 \pm 0.07$ \\
\hline
\end{tabular}

other sources in the literature and find them to be in agreement with their data.

Although we find an age- $\sigma$ trend for our sample of Coma early-type galaxies, the uncertainties in the age measurements are large and we cannot confirm a relation between these two parameters. Nonetheless, we observe that the low- $\sigma$ galaxies exhibit, on average, younger ages than their more massive counterparts.

In Paper I, we used the scatter in the color $-\sigma$ relation and the evolutionary stellar population synthesis models of Bruzual \& Charlot (2003) to estimate the formation epoch for our Coma galaxies. We found that, if we assume a strong coordination in the formation epoch of galaxies in the Coma cluster, most of these galaxies would have formed about $6 \mathrm{Gyr}$ ago and within a scatter of 1 Gyr. The results from our previous paper are consistent with those shown in the age- $\sigma$ panel of Figure 9. We find that the average age of low- $\sigma$ galaxies is $\sim 6$ Gyr and the scatter in the $\log \sigma-\log$ (age) relation implies a scatter in the formation epoch of $\sim 1.5 \mathrm{Gyr}$.

\subsection{Metallicity- $\sigma$}

We also find a trend between metallicity and $\log \sigma$ (shown in the middle panel of Figure 9). The high- $\sigma$ early-type galaxies tend to be more metal rich than the low- $\sigma$ galaxies which also exhibit a larger range in their metallicities.

Our derived slope, $[\mathrm{Z} / \mathrm{H}] \propto \sigma^{0.53 \pm 0.13}$, is in agreement with a number of studies which find a fairly robust metallicity- $\sigma$ relation (Kuntschner et al. 2001; Mehlert et al. 2003; Nelan et al. 2005; Thomas et al. 2005; Sánchez-Blázquez et al. 2006b; Bernardi et al. 2006). Furthermore, our metallicity $-\sigma$ trend is in good agreement with that of Nelan et al. (2005) which is also shown in Figure 9. We extend this trend to galaxies with $\sigma=$ $30 \mathrm{~km} \mathrm{~s}^{-1}$ with no evidence for a change of slope or offset.

\section{3. $[\alpha / F e]-\sigma$}

The $[\alpha / \mathrm{Fe}]$ also increases with increasing $\sigma$ (bottom panel in Figure 9). The low-mass early-type galaxies exhibit $\alpha$-ratios closer to the values in the solar neighborhood, although the trend is suggestive of $[\alpha / \mathrm{Fe}]>0$ even at the lowest $\sigma$. The higher mass galaxies have an overabundance of $[\alpha / \mathrm{Fe}]$.

A relation between $[\alpha / \mathrm{Fe}]$ and $\sigma$ has already been noted by a number of authors in the literature (Trager et al. 2000b; Kuntschner et al. 2001; Proctor \& Sansom 2002; Mehlert et al. 2003; Thomas et al. 2005; Bernardi et al. 2006), although conflicting with Proctor et al. (2004). In accordance with the former studies, we find that the $\alpha$-ratio increases with increasing velocity dispersion. However, due to large uncertainties, we can only confirm a trend and not a correlation between these parameters.

Our $[\alpha / \mathrm{Fe}]-\sigma$ slope of $0.17 \pm 0.07$ is shallower than the slopes derived by other authors who find $\sim 0.3$ (Trager et al. 2000b; Thomas et al. 2005; Nelan et al. 2005). However, we note that there are three objects in the $[\alpha / \mathrm{Fe}]-\sigma$ plot with $\alpha$-ratios that are quite large. These "outlying" points affect the average values of $[\alpha / \mathrm{Fe}]$ at a given $\sigma$ toward smaller values and therefore the slope. If we were to exclude these three galaxies (GMP 2306, GMP 3855, and GMP 3780), our slope would be $0.22 \pm 0.06$ which is consistent with other authors.

In general, supersolar $\alpha$-ratios denote that galaxies formed quickly, i.e., on short star formation timescales, and at high redshifts (Matteucci 1994). Therefore, an $[\alpha / \mathrm{Fe}]-\sigma$ trend suggests that the low- $\sigma$ galaxies had more extended star formation histories than their massive counterparts where new stars formed from already metal-enriched environment (Gorgas et al. 1997). In fact, a couple of mechanisms explaining the extended star formation histories of the low-mass galaxies already exist. One 

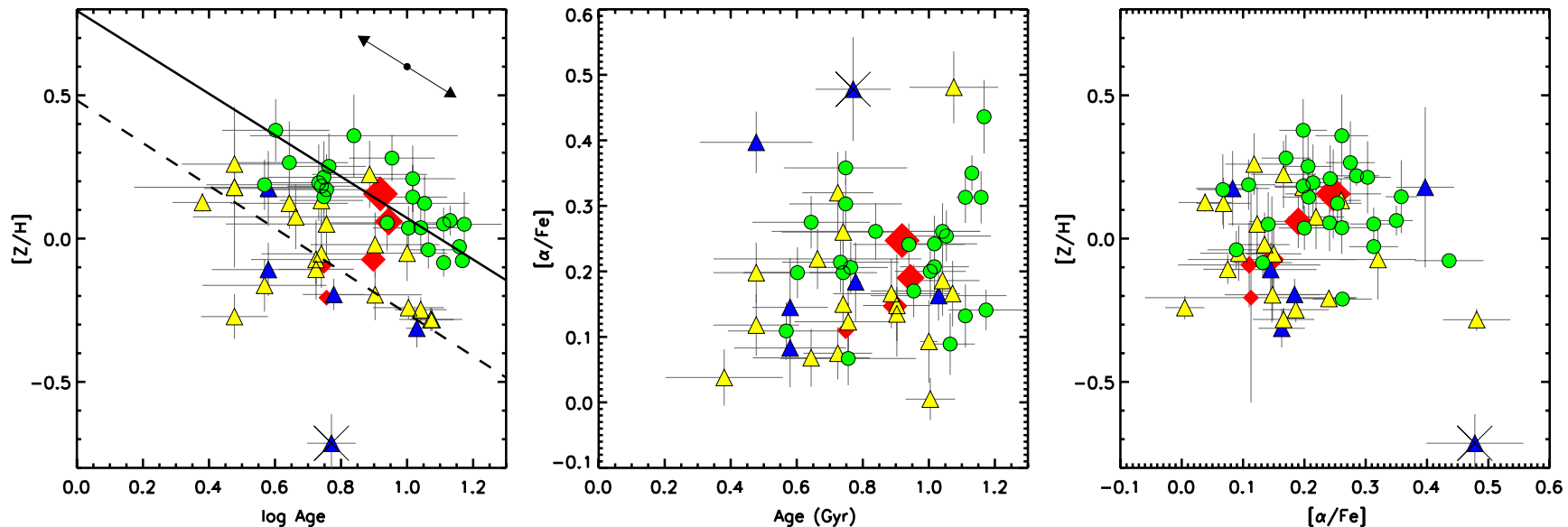

Figure 10. Metallicity vs. age, $[\alpha / \mathrm{Fe}]$ vs. age, and metallicity vs. $[\alpha / \mathrm{Fe}]$ for individual galaxies. The green circles represent galaxies with $100 \leqslant \sigma<185 \mathrm{~km} \mathrm{~s} \mathrm{~s}^{-1}$; the yellow triangles mark the galaxies with $50 \leqslant \sigma<100$; and the blue triangles are galaxies with $30 \leqslant \sigma<50$. The linear fit (left panel) for the high- $\sigma$ galaxies is marked with a solid line, while the fit to the low- $\sigma$ galaxies $\left(\sigma<100 \mathrm{~km} \mathrm{~s}^{-1}\right)$ is marked by a dashed line. We excluded the galaxy with the lowest metallicity from the linear regression (it is marked with a cross). The arrows in the top right corner of the age-metallicity plot represent the average correlated error ellipse for age and metallicity. The arrows in the age-metallicity plot represent $1 \sigma$ correlated error.

(A color version of this figure is available in the online journal.)

Table 10

Relations Between Age and Metallicity

\begin{tabular}{lccc}
\hline \hline$[\mathrm{Z} / \mathrm{H}]=a+b \cdot \log ($ age $)$ & Intercept & Slope & $\rho$ \\
\hline High- $\sigma$ & $0.795 \pm 0.143$ & $-0.723 \pm 0.128$ & -0.750 \\
Low- $\sigma$ & $0.482 \pm 0.105$ & $-0.744 \pm 0.119$ & -0.594
\end{tabular}

involves UV background radiation which can extend the duration of star formation by suppressing cooling more effectively in low-mass galaxies (Kawata 2001). While the other uses a combination of cooling, star formation, energy feedback, and chemical evolution to extend the star formation history of these galaxies (Chiosi \& Carraro 2002).

\section{TRENDS BETWEEN THE AGE, METALLICITY, AND $\alpha$-RATIO}

In Figure 10, we investigate relations between metallicity and age, $[\alpha / \mathrm{Fe}]$ and age, and metallicity and $[\alpha / \mathrm{Fe}]$ for our sample of Coma cluster early-type galaxies. We do not find any relations between the model parameters for all the galaxies in our sample. However, when we examine the difference between the highand low- $\sigma$ galaxies, we find trends in the age-metallicity and the metallicity $-[\alpha / \mathrm{Fe}]$ plots.

We find no correlation between age and $[\alpha / \mathrm{Fe}]$ or between metallicity and $[\alpha / \mathrm{Fe}]$ even at a fixed velocity dispersion. However, we do note a weak tendency for the high- $\sigma$ galaxies to have higher $\alpha$-ratios and to be more metal rich than the low- $\sigma$ galaxies. This effect is stronger in the Michielsen et al. (2007) sample (their Figure 7) whose data extend to lower metallicities and are in lower-density environment than our Coma cluster galaxies. Assuming that our and the Michielsen et al. (2007) sample span a similar range in $\sigma$, and that the metallicityabundance trends are not due to correlated errors, there is a possibility that this effect too is due to the environment.

The relation between the age and metallicity at a given $\sigma$ was first discussed by Trager et al. (2000a). We study the possibility of such relations for our low- and high- $\sigma$ galaxies. The Spearman-Rank coefficients (Table 10) imply that the relations between the age and the metallicity exist for the two $\sigma$-subsamples, while no correlations were found for either the age $-[\alpha / \mathrm{Fe}]$ nor the metallicity $-[\alpha / \mathrm{Fe}]$. Note that the one galaxy, marked by a cross in Figure 10, which we excluded from the Spearman-Rank test and linear regression is a galaxy with the lowest $\sigma=30 \mathrm{~km} \mathrm{~s}^{-1}$ in our sample.

Existence of an age-metallicity relation where galaxies with younger ages tend to be more metal rich, implies that these young galaxies have had multiple star formation episodes and that they had to form their stars from already enriched gas. This relation has been noted in numerous works both in lowdensity environments and clusters (Trager et al. 1998; Jørgensen 1999; Kuntschner 2000; Kuntschner et al. 2001; Poggianti et al. 2001a; Terlevich \& Forbes 2002; Sánchez-Blázquez et al. $2006 \mathrm{~b}$, to name a few). Some studies, however, suggest that this relation is a consequence of correlated errors (Trager et al. 1998, 2000a; Ferreras et al. 1999; Kuntschner et al. 2001). While Poggianti et al. (2001a) observe an age-metallicity relation at all magnitudes in the Coma cluster, Sánchez-Blázquez et al. (2006b) do not find this relation in Coma and argue that their apparent trend is due to correlated errors, although they also note a possibility that their sample is biased toward high- $\sigma$ galaxies, making the age-metallicity appear flat.

High- $\sigma$ galaxies follow the same relation as the agemetallicity relation found by Trager et al. (2000a). To investigate this, we estimated an average correlated error for our galaxies from the error ellipse derived with the iterative process as described in Section 6. The direction and size of this correlated error are shown in the top right corner of Figure 10. As can be seen, the direction of the correlated errors coincides with the slope of the age-metallicity relation. Furthermore, the size of the errors is consistent with the extent of the distribution of age and metallicity values. This implies that the age-metallicity correlation at a given velocity dispersion may be simply the result of correlated errors in both parameters.

The linear regression between the ages and metallicities for the low- and high- $\sigma$ galaxies shows that the slopes for the two subsamples are effectively the same, but offset with different zero points. At a given age, the high- $\sigma$ galaxies are more metal rich by a factor of $\sim 2$ than the low- $\sigma$ galaxies. Similarly, at a given metallicity, the low- $\sigma$ galaxies are $\sim 3 \mathrm{Gyr}$ younger than their more massive counterparts. This result also compares well with Michielsen et al. (2007) whose data sample contains 
$\mathrm{dE}$ galaxies from the Virgo cluster and the field. Both data sets are well anchored to the sample of massive Es from SánchezBlázquez et al. (2006b). However, there are differences in the distribution of galaxies between ours and their sample in the age-metallicity diagram (their Figure 7). The Coma cluster galaxies exhibit a similar range of ages to the Michielsen et al. (2007) dEs, but they have a smaller range in metallicity. This points toward an environmental dependence of the agemetallicity relation since the central region of the Coma cluster is one of the densest regions in the local universe. Unfortunately, we cannot say this with certainty without knowing how low in $\sigma$ the Michielsen et al. (2007) sample goes, since the one galaxy in our sample with very low metallicity on the age-metallicity plot is a galaxy with the lowest $\sigma=30 \mathrm{~km} \mathrm{~s}^{-1}$. The effect of finding the wider range in metallicity in the lower density environments than in the core of the Coma cluster, thus, may be purely due to sampling galaxies with lower-velocity dispersions.

\section{CONCLUSIONS}

In this paper, we study the properties of the underlying stellar populations of faint early-type galaxies in the core of the Coma cluster. Our sample is one of the largest homogeneous samples of cluster $\mathrm{dE} / \mathrm{dS} 0$ galaxies to date with velocity dispersions measurable down to $\sigma=30 \mathrm{~km} \mathrm{~s}^{-1}$. We present relations between 15 line-strength indices with $\sigma$ and extend them to the $\mathrm{dE} / \mathrm{dS} 0$ galaxies. We confirm that, when we include these faint early-type galaxies, the $\mathrm{Mg}_{2}$ relation and the $\mathrm{H} \beta$ $\sigma$ anticorrelation correspond well with other sources in the literature.

We find evidence for two types of behaviors between the metallic indices and $\sigma \mathrm{s}$. One set of indices $\left(\mathrm{C}_{2} 4668, \mathrm{Mg}_{1}, \mathrm{Mg}_{2}\right.$, $\left.\mathrm{Mg}_{b},[\mathrm{MgFe}]^{\prime}\right)$ exhibit tight linear relations with $\sigma$, which was also shown by other studies. The second group of I- $\sigma$ relations show a break in their slope where this break is more evident in a subset including $\mathrm{Ca} 4227, \mathrm{Fe} 5015, \mathrm{Fe} 5335,\langle\mathrm{Fe}\rangle$ than a subset with G4300, Fe4383, Ca4455, Fe4531, and Fe5270.

We also find that the relations between the Balmer lines and $\sigma$ have negative slopes and are fairly robust, with $\mathrm{H} \beta-\sigma$ having the weakest correlation coefficient. We find a hint of an asymmetric scatter in the $\mathrm{H} \beta-\sigma$ relation with more galaxies having a lower $\mathrm{H} \beta$ index. Since the asymmetry is in the opposite direction from that found in the lower density environments (Concannon et al. 2000), it is possible that this effect depends on the environment.

Although majority of the indices are influenced by the overall metallicity, we also investigated whether each $\mathrm{I}-\sigma$ group of indices is driven by the $\alpha$ - or Fe-peak elements. We found no connection between these elements and the occurrence of the break in the slope for the I- $\sigma$ trends. However, the nonBalmer indices with tight $\mathrm{I}-\sigma$ relations are all independent of microturbulent velocity of stellar atmospheres. This may be a main factor which determines the shape and/or tightness of the I $-\sigma$ relations for metal-dependent line strengths.

We use the SPMs to derive ages, metallicities, and $[\alpha / \mathrm{Fe}]$ for our Coma cluster galaxies. We find a wide range in all the SPM parameters where the galaxies with supersolar metallicities are dominated by the high- $\sigma$ galaxies, while the low- $\sigma$ galaxies are on average younger, have lower metallicities and their $\alpha$ ratios scatter around the solar value. This implies that the low- $\sigma$ galaxies had some residual star formation in their recent history, and that their star formation histories are more extended than they are for the high- $\sigma$ galaxies. These results are also confirmed by the trends we find between the age, metallicity, and $[\alpha / \mathrm{Fe}]$ with $\sigma$.
We find that the age-metallicity anticorrelation is most likely due to correlated errors. We were able to compare our results with those of Michielsen et al. (2007) who observed dE galaxies in the Virgo cluster and the field. Our Coma cluster galaxies seem to have a smaller range in metallicity when compared to the Michielsen et al. (2007) data set. Therefore, there is a possibility of an environmental effect on the metallicity range for $\mathrm{dE} / \mathrm{dS} 0$ galaxies, unless the Michielsen et al. (2007) data include galaxies with lower velocity dispersions.

We thank J. Cenarro for his help on investigating the spectra for potential problems and for providing the GC data. We are also grateful to Russell Smith for providing the data from the Hectospec Coma cluster survey and double checking our measurements. R. G. gratefully acknowledges University of Yale for the awarded nights at WIYN telescope. P. SánchezBlázquez acknowledges the support by a Marie Curie IntraEuropean Fellowship within the 6th European Community Framework Programme. We also thank the anonymous referee whose suggestions have improved this work.

\section{APPENDIX A}

\section{UNCERTAINTY MEASUREMENTS}

We created the "error spectra" which allowed us to measure the uncertainties in our line-strength measurements. This process consisted of multiple steps. First, we produced an optimal template for each galaxy by combining the six template stars (same procedure as in Paper I). The template stars are primarily $\mathrm{G}$ and $\mathrm{K}$ spectral type and are well matched with the dominating stellar population of early-type galaxies. Then, we shifted the optimal template of each galaxy to the rest frame and broadened this spectra and that of the galaxy to the resolution of Lick/IDS. Optimal template is a spectrum of a linear combination of template stars optimized to fit each galaxy spectrum (see Paper I for more details). We then found a polynomial fit (we chose the 7 th order polynomial) to the galaxy's and the template's blackbody curve. This allowed us to calculate the residuals between the galaxy spectrum and the "model galaxy" in the following way:

$$
R=G-\left(\frac{T}{P_{T}} \times P_{G}\right),
$$

where $R$ represents the residuals, $G$ the galaxy spectrum, $T$ the optimal template spectrum, $P_{G}$ the polynomial fit to the galaxy spectrum, and $P_{T}$ the polynomial fit to the template. The quantity in the parentheses is the "model galaxy," a spectrum with the exact shape of the galaxy and high $\mathrm{S} / \mathrm{N}$ features of the optimal template. $R$ refers to the residual noise between the galaxy spectrum and the model galaxy.

However, to build a true error spectrum we must take into account the actual noise of the galaxy together with the uncertainty due to the template mismatch. We did this by taking the square root of the galaxy and scaling this spectrum to the average number of its counts. Then, we multiplied that quantity by the amount of noise from the residuals:

$$
E=\frac{\sqrt{G}}{\langle\sqrt{G}\rangle} \times \sqrt{\left\langle R^{2}\right\rangle}
$$

Here, $E$ is the error spectrum, $\langle\sqrt{G}\rangle$ is the average number of counts of the square root of the galaxy, and $\left\langle R^{2}\right\rangle$ is the average number of counts of residuals squared. The error 
spectrum created in this way is, in a sense, a spectrum of the exact same shape as the galaxy with the level of noise which takes into account the Poissonian noise and the uncertainty due to the mismatch between the templates and the galaxy spectrum. Additionally, we calculate the uncertainty associated with the flux calibration. The program INDEX estimates the uncertainties associated with flux calibration by measuring the line-strength indices with all the individual response curves and computing the standard deviation from these measurements. In summary, our uncertainty measurements include the photon noise, the error due to the mismatch between the galaxy and the template, and the flux-related error.

\section{APPENDIX B}

\section{DETERMINING SIGNAL-TO-NOISE RATIO}

In our study of both the internal kinematics and stellar populations of the faint early-type galaxies, we consider only the galaxies whose average $S / N \geqslant 15$ (see Paper I). There are 74 galaxies in our central Coma cluster sample which satisfy this condition. The $\mathrm{S} / \mathrm{N}$ which we calculate for our galaxies is an average value of $\mathrm{S} / \mathrm{N}$ per pixel.

Determining the $\mathrm{S} / \mathrm{N}$ involved a couple of steps. A few of these steps are already described in Appendix A, where we also show how one finds the residuals (Equation A1) between the galaxy spectrum and the model galaxy. We define an average value of $\mathrm{S} / \mathrm{N}$ per pixel of each galaxy as

$$
\mathrm{S} / \mathrm{N}=\frac{\langle G\rangle}{\sqrt{R^{2}}} .
$$

The value of $\mathrm{S} / \mathrm{N}$ calculated in this way is an average value for each galaxy and it includes the mismatch between the actual galaxy spectrum and that of the model galaxy.

\section{REFERENCES}

Bender, R., Burstein, D., \& Faber, S. M. 1993, ApJ, 411, 153

Bernardi, M., Nichol, R. C., Sheth, R. K., Miller, C. J., \& Brinkmann, J. 2006, AJ, 131, 1288

Bernardi, M., Renzini, A., da Costa, L. N., Wegner, G., Alonso, M. V., Pellegrini, P. S., Rité, C., \& Willmer, C. N. A. 1998, ApJ, 508, L143

Brodie, J. P., \& Huchra, J. P. 1991, ApJ, 379, 157

Bruzual, G., \& Charlot, S. 2003, MNRAS, 344, 1000

Burstein, D., Faber, S. M., Gaskell, C. M., \& Krumm, N. 1984, ApJ, 287, 586

Caldwell, N. 1983, AJ, 88, 804

Caldwell, N., Rose, J. A., \& Concannon, K. D. 2003, AJ, 125, 2891

Caldwell, N., Rose, J. A., Sharples, R. M., Ellis, R. S., \& Bower, R. G. 1993, AJ, 106,473

Cardiel, N. 1999, PhD thesis, Universidad Complutense de Madrid, Spain

Cenarro, A. J., Beasley, M. A., Strader, J., Brodie, J. P., \& Forbes, D. A. 2007, AJ, 134,391

Chiosi, C., \& Carraro, G. 2002, MNRAS, 335, 335

Colless, M., Burstein, D., Davies, R. L., McMahan, R. K., Saglia, R. P., \& Wegner, G. 1999, MNRAS, 303, 813

Colless, M., \& Dunn, A. M. 1996, ApJ, 458, 435

Concannon, K. D., Rose, J. A., \& Caldwell, N. 2000, ApJ, 536, L19

Denicoló, G., Terlevich, R., Terlevich, E., Forbes, D. A., \& Terlevich, A. 2005, MNRAS, 358, 813
Dressler, A. 1984, ApJ, 281, 512

Ferguson, H. C., \& Binggeli, B. 1994, A\&AR, 6, 67

Ferreras, I., Charlot, S., \& Silk, J. 1999, ApJ, 521, 81

Fisher, D., Franx, M., \& Illingworth, G. 1995, ApJ, 448, 119

Forbes, D. A., Ponman, T. J., \& Brown, R. J. N. 1998, ApJ, 508, L43

González, J. D. J. 1993, PhD thesis, Univ. California, Santa Cruz

Gorgas, J., Efstathiou, G., \& Aragon Salamanca, A. 1990, MNRAS, 245, 217

Gorgas, J., Faber, S. M., Burstein, D., Gonzalez, J. J., Courteau, S., \& Prosser, C. 1993, ApJS, 86, 153

Gorgas, J., Pedraz, S., Guzman, R., Cardiel, N., \& Gonzalez, J. J. 1997, ApJ, 481, L19

Graham, A. W., \& Guzmán, R. 2003, AJ, 125, 2936

Greggio, L. 1997, MNRAS, 285, 151

Guzman, R., Lucey, J. R., Carter, D., \& Terlevich, R. J. 1992, MNRAS, 257 187

Hammer, F., Gruel, N., Thuan, T. X., Flores, H., \& Infante, L. 2001, ApJ, 550, 570

Jorgensen, I. 1997, MNRAS, 288, 161

Jørgensen, I. 1999, MNRAS, 306, 607

Jorgensen, I., Franx, M., \& Kjaergaard, P. 1996, MNRAS, 280, 167

Kawata, D. 2001, ApJ, 558, 598

Kelson, D. D., Illingworth, G. D., Franx, M., \& van Dokkum, P. G. 2006, ApJ, 653,159

Korn, A. J., Maraston, C., \& Thomas, D. 2005, A\&A, 438, 685

Kuntschner, H. 2000, MNRAS, 315, 184

Kuntschner, H., \& Davies, R. L. 1998, MNRAS, 295, L29

Kuntschner, H., Lucey, J. R., Smith, R. J., Hudson, M. J., \& Davies, R. L. 2001, MNRAS, 323, 615

Kuntschner, H., Smith, R. J., Colless, M., Davies, R. L., Kaldare, R., \& Vazdekis, A. 2002, MNRAS, 337, 172

Maraston, C. 1998, MNRAS, 300, 872

Matković, A., \& Guzmán, R. 2005, MNRAS, 362, 289

Matteucci, F. 1994, A\&A, 288, 57

Mehlert, D., Thomas, D., Saglia, R. P., Bender, R., \& Wegner, G. 2003, A\&A, 407, 423

Michielsen, D., et al. 2008, MNRAS, 385, 1374

Nelan, J. E., Smith, R. J., Hudson, M. J., Wegner, G. A., Lucey, J. R., Moore, S. A. W., Quinney, S. J., \& Suntzeff, N. B. 2005, ApJ, 632, 137

Pedraz, S, Gorgas, J, Cardiel, N., \& Guzmán, R. 1998, Ap\&SS, 632, 1374

Poggianti, B. M., et al. 2001a, ApJ, 562, 689

Poggianti, B. M., et al. 2001b, ApJ, 563, 118

Proctor, R. N., Forbes, D. A., Hau, G. K. T., Beasley, M. A., De Silva, G. M., Contreras, R., \& Terlevich, A. I. 2004, MNRAS, 349, 1381

Proctor, R. N., \& Sansom, A. E. 2002, MNRAS, 333, 517

Rakos, K., \& Schombert, J. 2004, AJ, 127, 1502

Sánchez-Blázquez, P., Gorgas, J., Cardiel, N., \& González, J. J. 2006a, A\&A, 457,787

Sánchez-Blázquez, P., Gorgas, J., Cardiel, N., \& González, J. J. 2006b, A\&A, 457,809

Smith, R. J., et al. 2008, MNRAS, 386, L96

Terlevich, R., Davies, R. L., Faber, S. M., \& Burstein, D. 1981, MNRAS, 196 381

Terlevich, A. I., \& Forbes, D. A. 2002, MNRAS, 330, 547

Terlevich, A. I., Kuntschner, H., Bower, R. G., Caldwell, N., \& Sharples, R. M. 1999, MNRAS, 310, 445

Thomas, D., Maraston, C., \& Bender, R. 2002, Ap\&SS, 281, 371

Thomas, D., Maraston, C., \& Bender, R. 2003, MNRAS, 343, 279

Thomas, D., Maraston, C., Bender, R., \& Mendes de Oliveira, C. 2005, ApJ, 621,673

Thomas, D., Maraston, C., \& Korn, A. 2004, MNRAS, 351, L19

Trager, S. C., Faber, S. M., Worthey, G., \& González, J. J. 2000a, AJ, 120, 165

Trager, S. C., Faber, S. M., Worthey, G., \& González, J. J. 2000b, AJ, 119, 1645

Trager, S. C., Worthey, G., Faber, S. M., Burstein, D., \& Gonzalez, J. J. 1998, ApJS, 116, 1

Tripicco, M. J., \& Bell, R. A. 1995, AJ, 110, 3035

Worthey, G. 1994, ApJS, 95, 107

Worthey, G., \& Collobert, M. 2003, ApJ, 586, 17

Worthey, G., Faber, S. M., \& Gonzalez, J. J. 1992, ApJ, 398, 69

Worthey, G., Faber, S. M., Gonzalez, J. J., \& Burstein, D. 1994, ApJS, 94, 687

Worthey, G., \& Ottaviani, D. L. 1997, ApJS, 111, 377 